\title{
IDENTITIES ON QUADRATIC GAUSS SUMS
}

\author{
PAUL GÉRARDIN AND WEN-CH' ING WINNIE LI
}

\begin{abstract}
Given a local field $F$, each multiplicative character $\theta$ of the split algebra $F \times F$ or of a separable quadratic extension of $F$ has an associated generalized Gauss sum $\gamma_{\theta}^{F}$. It is a complex valued function on the character group of $F^{\times} \times F$, meromorphic in the first variable. We define a pairing between such Gauss sums and study its properties when $F$ is a nonarchimedean local field. This has important applications to the representation theory of $G L(2, F)$ and correspondences [GL3].
\end{abstract}

\section{INTRODUCTION}

The multiplicative group $F^{\times}$of a local field $F$ is a split extension of the value group $\left|F^{\times}\right|$by the compact group of the units. Hence, the group $\mathscr{A}\left(F^{\times}\right)$ of continuous homomorphisms of $F^{\times}$in $\mathbf{C}^{\times}$is a one-dimensional complex Lie group, with connected component of identity the image of $\mathbf{C}$ under the map

$$
s \mapsto\left(t \mapsto|t|^{s}\right) .
$$

We have written $|t|$ for the normalized absolute value of $t$.

The group $F^{\times}$acts on functions on $F$ by translations:

$$
t: f \mapsto f^{t}, \quad f^{t}(x)=f(t x), \quad t \in F^{\times} .
$$

This gives an action of $F^{\times}$on the space $\mathscr{S}(F)$ of Schwartz-Bruhat functions on $F$, hence also an action on the space $\mathscr{S}^{\prime}(F)$ of tempered distributions on $F$ :

$$
\left\langle t . D \mid f^{t}\right\rangle=\langle D \mid f\rangle .
$$

For each $\chi$ in $\mathscr{A}\left(F^{\times}\right)$, the space of tempered distributions of type $\chi$ under the action of $F^{\times}$is one-dimensional (e.g. [W3]). The choice of a nontrivial additive unitary character $\psi$ of $F$ defines an identification of $F$ with its Pontrjagin dual by $(u, v) \mapsto \psi(u v)$, hence a self-dual Haar measure $d_{\psi} u$ on $F$, and a Fourier transform

$$
\hat{f}(v)=\int_{F} f(u) \psi(u v) d_{\psi} u, \quad f \in \mathscr{S}(F) .
$$

Received by the editors July 10, 1988 and, in revised form, November 21, 1988 .

1980 Mathematics Subject Classification (1985 Revision). Primary 11L05; Secondary 11S37, $33 \mathrm{~A} 15$.

Key words and phrases. Gauss sum, quadratic gamma factor.

Both authors supported in part by NSF Grant DMS-8404083. 
The Fourier transform exchanges distributions of type $\chi$ with distributions of type $t \mapsto|t| \chi(t)^{-1}$.

Fix an additive Haar measure $d u$ on $F$ and let $d^{*} u$ be the measure $|u|^{-1 / 2} d u$ on $F$. Then, for each $\chi \in \mathscr{A}\left(F^{\times}\right)$, we have a measure $\chi d^{*}$ on $F^{\times}$, which is holomorphic in $\chi$. It is well known [W3] that $\chi d^{*}$ extends to a unique meromorphic distribution $\Delta_{\chi}$ of type $\left.\chi\right|^{1 / 2}$ on $F$, which has simple poles. As $\hat{\Delta}_{\chi}$ is a multiple of $\Delta_{\chi^{-1}}$, denote their ratio by $\gamma^{F}(\chi, \psi)$, called the gamma factor attached to $\chi$ and $\psi$ :

$$
\hat{\Delta}_{\chi}=\gamma^{F}(\chi, \psi) \Delta_{\chi^{-1}} \text {. }
$$

It is a meromorphic function of $\chi$, satisfying the complement formula

$$
\gamma^{F}(\chi, \psi) \gamma^{F}\left(\chi^{-1}, \psi^{-1}\right)=1,
$$

and also

$$
\begin{aligned}
& \gamma^{F}\left(\chi, \psi^{t}\right)=\chi(t)^{-1} \gamma^{F}(\chi, \psi), \quad t \in F^{\times}, \\
& \gamma^{F}(1, \psi)=1 .
\end{aligned}
$$

In case $F$ is a nonarchimedean local field, the gamma factor $\gamma^{F}(\chi, \psi)$ is a Gauss sum; more precisely, it is an analytic continuation of the following integral taken in principal value:

$$
\int_{F} \chi(u) \psi(u) d_{\psi}^{*} u
$$

Let $K$ be a quadratic étale algebra over $F$, that is, $K$ is either an $F$-algebra isomorphic (in two ways) to $F \times F$, or a separable quadratic field extension of $F$. Then the norm group $N_{K / F}\left(K^{\times}\right)$has index 1 or 2 according as $K$ splits or not over $F$. Denote by $\eta_{K / F}$ the character of $F^{\times}$with kernel $N_{K / F}\left(K^{\times}\right)$. For a character $\theta$ of $K^{\times}$, a character $\chi$ of $F^{\times}$, and $\psi$ as above, we define the quadratic Gauss sum with $T_{K / F}$ the trace form from $K$ to $F$ :

$$
\gamma_{\theta}^{F}(\chi, \psi)=\lambda_{K / F}(\psi) \gamma^{K}\left(\theta \chi \circ N_{K / F}, \psi \circ T_{K / F}\right),
$$

where

$$
\lambda_{K / F}(\psi)=\gamma^{F}\left(\eta_{K / F}, \psi\right) .
$$

Note that in case $K$ is split over $F$, the group $\mathscr{A}\left(K^{\times}\right)$is isomorphic to the product of two copies of $\mathscr{A}\left(F^{\times}\right)$so that we may identify $\theta$ with a couple $\{\mu, \nu\}$ of characters of $F^{\times}$; in this case, we have $\lambda_{K / F}(\psi)=1$, and

$$
\gamma_{\theta}^{F}(\chi, \psi)=\gamma^{F}(\chi \mu, \psi) \gamma^{F}(\chi \nu, \psi) .
$$

It is known that these quadratic Gauss sums have the following properties:

(a) if the additive character $\psi$ is changed to $\psi^{t}, t \in F^{\times}$, then

$$
\gamma_{\theta}^{F}\left(\chi, \psi^{t}\right)=\chi(t)^{-2} \omega(t)^{-1} \gamma_{\theta}^{F}(\chi, \psi), \quad \omega(t)=\eta_{K / F}(t) \theta(t) ;
$$


(b) it satisfies the Davenport-Hasse identity, namely, when $\theta=\mu \circ N_{K / F}$ for some character $\mu$ of $F^{\times}$, one has

$$
\gamma_{\theta}^{F}(\chi, \psi)=\gamma^{F}(\chi \mu, \psi) \gamma^{F}\left(\chi \mu \eta_{K / F}, \psi\right) ;
$$

(c) for $F$ nonarchimedean and $\chi$ of conductor large enough

$$
\gamma_{\theta}^{F}(\chi, \psi)=\gamma^{F}(\chi, \psi) \gamma^{F}(\chi \omega, \psi),
$$

where $\omega$ is as in (a); this is the deep twist property.

In this article, we shall study, for $F$ nonarchimedean, a pairing between two such quadratic Gauss sums $\gamma_{\theta}^{F}$ and $\gamma_{\theta^{\prime}}^{F}$ relative to two quadratic étale algebras $K$ and $K^{\prime}$. It is defined for the two meromorphic functions

$$
\chi \mapsto \gamma_{\theta}^{F}(\chi, \psi) \text { and } \chi \mapsto \gamma_{\theta^{\prime}}^{F}\left(\chi^{-1}, \psi\right)
$$

having no common pole as the finite part of a contour integral over $\mathscr{A}\left(F^{\times}\right)$ enclosing the poles of $\gamma_{\theta^{\prime}}^{F}\left(\chi^{-1}, \psi\right)$ but no poles of $\chi \mapsto \gamma_{\theta}^{F}(\chi, \psi)$ :

$$
\left\langle\gamma_{\theta}^{F} \mid \gamma_{\theta^{\prime}}^{F}\right\rangle_{\psi}=\oint_{\mathscr{A}\left(F^{\times}\right)} \gamma_{\theta}^{F}(\chi, \psi) \gamma_{\theta^{\prime}}^{F}\left(\chi^{-1}, \psi\right) d \chi .
$$

Our purpose is to derive further properties of the quadratic Gauss sums from this pairing, with the goal of reestablishing Langland's correspondences on representations of degree two semisimple algebras over $F$. Thus, some of the results would become immediate consequences if one were to grant these correspondences.

Our main result (Theorem 1) expresses the value of this pairing in terms of a gamma factor coming from the étale $F$-algebra $B=K \otimes_{F} K^{\prime}$ and the character $\theta \times \theta^{\prime}$ of $B^{\times}$defined by

$$
\left(\theta \times \theta^{\prime}\right)(z)=\theta \circ N_{B / K}(z) \theta^{\prime} \circ N_{B / K^{\prime}}(z):
$$

In Theorem 2, we show that for $K^{\prime}$ split the formula reduces to a formula which has appeared already in [L, GL1, GL2], called the multiplicative formula for $\gamma_{\theta}^{F}$. When $K$ and $K^{\prime}$ are not isomorphic and when the product of the restriction to $F^{\times}$of $\theta$ and $\theta^{\prime}$ is the character $t \mapsto|t|^{-1}$, the value of the pairing $\left\langle\gamma_{\theta}^{F} \mid \gamma_{\theta^{\prime}}^{F}\right\rangle_{\psi}$ can be simplified (§3.3). In particular, it depends only on the fields $K$ and $K^{\prime}$. This fact is used in [GL3] to characterize the degree two monomial representations of the local Weil group $W_{F}$ over $F$. Each character $\theta$ of $K^{\times}$determines a two-dimensional representation $\operatorname{Ind}_{K}^{F} \theta$ of $W_{F}$. We prove in $\S 3.4$ that the quadratic Gauss sums $\gamma_{\theta}^{F}$ parametrize the isomorphism classes of these representations $\operatorname{Ind}_{K}^{F} \theta$.

\section{Preparation}

1.1. We introduce some notations for any nonarchimedean field. In general, the field will be indicated by a subscript, but it will be deleted for the base field 
$F$. The ring of integers of $F$ is $\mathscr{O}=\mathscr{O}_{F}$, its group of units is $\mathscr{O}^{\times}=\mathscr{O}_{F}^{\times}$, its maximal ideal is $\mathscr{P}=\mathscr{P}_{F}$. As $\mathscr{O}$ and $\mathscr{O}^{\times}$are open compact subgroups of $F$, $F^{\times}$, respectively, we choose Haar measures $d u=d_{F} u$ on $F, d^{\times} t=d_{F}^{\times} t$ on $F^{\times}$, respectively, giving to them the volume 1 . Then $d^{\times} t=L_{F}|t|^{-1} d t$, where $L_{F}=\left(1-q^{-1}\right)^{-1}$ is the value at the character $t \rightarrow|t|$ of the $L$-function of $F$, and $q=q_{F}$ is the module of $F$.

On the group $\widehat{F}$ of characters of $F$, there is an absolute value || defined on $\psi \in \widehat{F}$ as the smallest number $c$ in the value group of $F$ such that $\psi(u)=1$ for $c|u| \leq 1$. Then $\left|\psi^{t}\right|=|\psi||t|$ for $t$ in $F$. The self-dual Haar measure on $F$ associated to the bicharacter $\psi(u v)$, for $\psi$ nontrivial in $\widehat{F}$, is $d_{\psi} u=$ $|\psi|^{1 / 2} d u$.

We define a modification $\Gamma^{F}=\Gamma$ of the gamma factor $\gamma^{F}$ by taking the finite part of the following integral:

$$
\Gamma(\chi, \psi)=\int_{F} \chi(t) \psi(t) d^{\times} t
$$

It is given in terms of $\gamma^{F}$ by

$$
\Gamma(\chi, \psi)=L_{F}|\psi|^{-1 / 2} \gamma^{F}\left(\chi q^{1 / 2}, \psi\right)
$$

and satisfies the following complement formula:

$$
\Gamma(\chi, \psi) \Gamma\left(\chi^{-1} q^{-1}, \psi\right)=L_{F}^{2}|\psi|^{-1} \chi(-1) .
$$

Here, we have used the convention which identifies a nonzero complex number $Z$ with the character $t \mapsto Z^{\text {ord } t}$ of $F^{\times}$, where ord $t=-\log _{q}|t|$.

For a character $\chi$ of $F^{\times}$, we write $a(\chi)$ for its conductor, and $A(\chi)=q^{a(\chi)}$; so, $A(\chi)$ is the smallest number $c \geq 1$ such that, for $t$ a unit, we have $\chi(t)=1$ when $c|t-1| \leq 1$, i.e. $\operatorname{ord}(t-1) \geq a(\chi)$ means $|t-1| A(\chi) \leq 1$. We define also $A^{\prime}(\chi)$ to be $A(\chi)$ if $\chi$ ramifies and to be $q$ otherwise. We denote by $|\chi|$ the character $t \mapsto|\chi(t)|$, so that it coincides with $|Z|$ when $\chi$ is given by the nonzero complex number $Z$.

For $|\chi|<q^{1 / 2}$ the gamma factor $\gamma^{F}(\chi, \psi)$ is given by the convergent integral

$$
\gamma^{F}(\chi, \psi)=\int_{|t| \leq A^{\prime}(\chi)|\psi|^{-1}} \chi(t) \psi(t) d_{\psi}^{*} t,
$$

with $d_{\psi}^{*} t=|t|^{-1 / 2} d_{\psi} t$. When $\chi$ ramifies, only the shell $|t|=A(\chi)|\psi|^{-1}$ contributes; moreover, there is $\chi_{\psi}$ in this shell such that, for any character $\mu$ of $F^{\times}$satisfying $A(\mu)^{2} \leq A(\chi)$,

$$
\gamma^{F}(\chi \mu, \psi)=\mu\left(\chi_{\psi}\right) \gamma^{F}(\chi, \psi)
$$

From this, it follows that for $K=F \times F$ and $\theta$ a character of $K^{\times}$given by the characters $\mu$ and $\nu$ of $F^{\times}$, we have the relation

$$
\gamma_{\theta}^{F}(\chi, \psi)=\gamma^{F}(\chi, \psi) \gamma^{F}(\chi \omega, \psi) \quad \text { if } A(\mu)^{2} \text { and } A(\nu)^{2} \leq A(\chi), \quad \omega=\mu \nu .
$$


1.2. For $K$ an étale $F$-algebra, that is, a product of the extensions $E$ of $F$, its ring of integers $\mathscr{O}_{K}$ is the product of the $\mathscr{O}_{E}$ 's, and the group $\mathscr{O}_{K}^{\times}$of units is the product of the $\mathscr{O}_{E}^{\times}$'s. Let $T=T_{K / F}$ be the trace form from $K$ to $F$. Then the pairing $(x, y) \mapsto T(x y)$ from $K \times K$ to $F$ is nondegenerate. By composition with $F \rightarrow F / \mathscr{O}_{F}$, we get an orthogonality relation between the $\mathscr{O}_{K^{-}}$submodules of $K$. The index of $\mathscr{O}_{K}$ in its orthogonal is called the discriminant $D_{K / F}$ : it is the product of the $D_{E / F}$ 's. The self-dual Haar measure $d_{K, \psi}$ on $K$ associated to the bicharacter $\psi \circ T(x y)$ is $|\psi \circ T|^{1 / 2} d_{K}$, with $|\psi \circ T|$ the product of the $\left|\psi \circ T_{E / F}\right|$ 's; hence $d_{K, \psi}$ is the product of the $d_{E, \psi}$ 's. From Corollary 3 to Proposition 4 of Chapter VIII-1 in [W2], we have $|\psi \circ T|=|\psi|^{[K: F]} D_{K / F}^{-1}$. Let $N=N_{K / F}$ be the norm map on $K$ : it is the product of the norm maps $N_{E / F}$. We introduce the two numbers

$$
L_{K}=\prod_{E} L_{E}=\prod_{E}\left(1-q_{E}^{-1}\right)^{-1} \quad \text { and } \quad L_{K / F}=L_{K} / L_{F}^{[K: F]} .
$$

Note that $L_{K}$ is also the integral over $\mathscr{O}_{K}$ of the function $|N x|$ for the measure $d_{K}^{\times}$. We denote by $|x|_{K}$ the absolute value $\operatorname{Max}_{E}\left|N_{E / F} x_{E}\right|$ on $K, x=\left(x_{E}\right)$.

1.3. A quadratic étale $F$-algebra $K$ has a conjugation ${ }^{-}$; its norm $N=N_{K / F}$ and trace $T=T_{K / F}$ are also given by $N x=x \bar{x}, T x=x+\bar{x}$. For each nontrivial additive character $\psi$ of $F$, the quadratic character $\psi \circ N$ is nondegenerate and defines a fourth roots of unity $\lambda(\psi \circ N)$ by the functional equation [W1, G]:

$$
\int_{K} \hat{f}(y) \psi \circ N(y) d y=\lambda(\psi \circ N) \int_{K} f(x) \psi^{-1} \circ N(x) d x
$$

where $f$ lies in the Schwartz-Bruhat space $\mathscr{S}(K)$ of compactly supported locally constant functions on $K$, and its Fourier transform is

$$
\hat{f}(y)=\int_{K} f(x) \psi \circ T(x \bar{y}) d_{K, \psi} y .
$$

With $f$ the characteristic function of a sufficiently small ball around 0 , we get

$$
\lambda(\psi \circ N)=\int_{|x|_{K} \leq R} \psi \circ N(x) d_{K, \psi} x, \quad \text { for } R \text { large enough . }
$$

Moreover, decomposing $F$ with respect to the norm group of $K$, we get

$$
\lambda(\psi \circ N)=\gamma^{F}\left(\eta_{K / F}, \psi\right),
$$

with the notations as in the introduction. Note that $\lambda(\psi \circ N)=1$ for $K$ split.

1.4. If $K$ is a quadratic étale $F$-algebra and $\theta$ is a character of $K^{\times}$, we define a two-dimensional representation of the Weil group $W_{F}$ of $F$ as follows. If $K$ a field, its Weil group $W_{K}$ appears as the kernel of the composition of the class field theory map $W_{F} \rightarrow F^{\times}$with $\eta_{K / F}$; it has index two in $W_{F}$ and $\theta$ gives a one-dimensional representation of $W_{K}$ from the map $W_{K} \rightarrow K^{\times}$, hence by 
induction a two-dimensional representation $\operatorname{Ind}_{K}^{F} \theta$ of $W_{F}$. If $K$ is split, then $\theta$ is given by two characters $\mu$ and $\nu$ of $F^{\times}$; in this case $\operatorname{Ind}_{K}^{F} \theta$ is the sum of the two one-dimensional representations of $W_{F}$ defined by $\mu$ and $\nu$.

Lemma. Assume that $K$ is a separable quadratic extension of $F$. Given a character $\theta$ of $K^{\times}$and two characters $\alpha$ and $\beta$ of $F^{\times}$, the following conditions are equivalent:

(i) $\theta=\alpha \circ N_{K / F}=\beta \circ N_{K / F}, \beta=\alpha \eta_{K / F}$,

(ii) $\operatorname{Ind}_{K}^{F} \theta=\alpha \oplus \beta$,

(iii) $\gamma_{\theta}^{F}(\chi, \psi)=\gamma^{F}(\chi \alpha, \psi) \gamma^{F}(\chi \beta, \psi)$ for any character $\chi$ of $F^{\times}$.

Proof. (i) $\Rightarrow$ (ii) is clear. (ii) $\Rightarrow$ (i) comes from the fact that $\operatorname{Ind}_{K}^{F} \theta$ is reducible if and only if the character $\theta$ is fixed under the conjugation of $K$ over $F$. (i) $\Rightarrow$ (iii) is the Davenport-Hasse identity. Finally (iii) $\Rightarrow$ (i) uses the fact that $\theta$ is not regular since $\gamma_{\theta}^{F}$ has poles, so $\theta=\alpha \circ N_{K / F}$ and $\gamma_{\theta}^{F}(\chi, \psi)$ is $\gamma^{F}(\chi \alpha, \psi) \gamma^{F}\left(\chi \alpha \eta_{K / F}, \psi\right)$ with poles at $\alpha^{-1} q^{-1 / 2}$ and $\alpha^{-1} \eta_{K / F} q^{-1 / 2}$, which are also $\alpha^{-1} q^{-1 / 2}$ and $\beta^{-1} q^{-1 / 2}$ : this gives the implication.

1.5. Lemma. Let $K$ be a quadratic $F$-algebra, with norm $N$, trace $T$, discriminant $D$, absolute value ||$_{K}$. Fix a nontrivial additive character $\psi$ of $F$. If the element $R$ of $\left|K^{\times}\right|_{K}$ satisfies $R|\psi| \geq D$, then, the Fourier transform with respect to $\psi \circ T$ of the function $g$ on $K$ defined by

$$
g(x)=\psi(-N x) \text { for }|x-1|_{K} \leq R \text { and } 0 \text { otherwise }
$$

is equal to $\lambda_{K / F}(\psi)^{-1} \bar{g}$.

Proof. We write the inequality $R|\psi| \geq D$ as $R^{-1}|\psi \circ T|^{-1}$; this gives $\psi(N x)=$ 1 for $|x|_{K} \leq R^{-1}|\psi \circ T|^{-1}$; the orthogonal of this subgroup with respect to the bicharacter $\psi \circ T(x \bar{y})$ is $|y|_{K} \leq R$. From the reduction theorem in [G], the $\lambda$-factor of the quadratic group $(K, \psi \circ N)$ is also the $\lambda$-factor of the factor group of the ideal $|x|_{K} \leq R$ modulo the ideal $|x|_{K} \leq R^{-1}|\psi \circ T|^{-1}$ for the quadratic character $\psi \circ N$, hence

$$
\lambda_{K / F}(\psi) \int_{|x|_{K} \leq R} f(x) \psi \circ N(x)^{-1} d x=\int_{|x|_{K} \leq R} \hat{f}(y) \psi \circ N(y) d y,
$$

for all $f$ in $\mathscr{S}(K)$. We write now the value at $1+z$ of the Fourier transform of the given function $g: \hat{g}(1+z)=\psi(1+T z) \int_{|x|_{K} \leq R} \psi(-N x+T x z) d_{K, \psi} x$. We apply then the above formula to the function $f(x)=\psi(T x z)$ for $|x|_{K} \leq R$ and 0 elsewhere:

$$
\hat{g}(1+z)=\psi(1+T z) \lambda_{K / F}(\psi)^{-1} \int_{\substack{|x-z|_{K} \leq R^{-1}|x|_{K} \leq R \\|x|^{-1}}} \psi(N x) d_{K, \psi} x \int_{|y|_{K} \leq R} d_{K, \psi} y .
$$

By integrating first on the ball $|x|_{K} \leq R^{-1}|\psi \circ T|^{-1}$, the first integral is seen to be 0 unless $|z|_{K} \leq R$; in this case, we get

$$
\hat{g}(1+z)=\psi(1+T z) \lambda_{K / F}(\psi)^{-1} \psi(N z)=\lambda_{K / F}(\psi)^{-1} \psi \circ N(1+z),
$$

which proves the lemma. 
1.6. Biquadratic étale $F$-algebras. A biquadratic étale $F$-algebra $B$ is a fourdimensional étale $F$-algebra containing at least two quadratic sub- $F$-algebras $K$ and $K^{\prime}$. Then, the map $(x, y) \mapsto x y$ gives an isomorphism from $K \otimes_{F} K^{\prime}$ onto $B$. The conjugation of $K$ (resp. $K^{\prime}$ ) with respect to $F$ extends to an $F$-involution on $B$ with $K^{\prime}$ (resp. $K$ ) as fixed points. These two involutions commute, and their composition has fixed points a third quadratic subalgebra $K^{\prime \prime}$, and $K, K^{\prime}, K^{\prime \prime}$ are all the three quadratic sub- $F$-algebras in $B$. When $B$ is a field, it is a biquadratic extension of $F$, and $K, K^{\prime}, K^{\prime \prime}$ are the three quadratic extensions of $F$ contained in $B$. When $B$ contains exactly one split quadratic $F$-algebra, it is a direct product of two isomorphic quadratic separable extensions of $F$ : we see $B$ as $K \times K$ with the two subfields $\{(x, x) \mid x \in K\}$, $\{(x, \bar{x}) \mid x \in K\}$ and the split algebra $F \times F$ embedded naturally; in this case, the three involutions are respectively $(x, y) \mapsto(y, x),(\bar{y}, \bar{x}),(\bar{x}, \bar{y})$, we have isomorphisms from $K \otimes_{F} K$ onto $B$ and from $K \otimes_{F}(F \times F)$ onto $B$ given by $x \otimes y \mapsto(x y, x \bar{y})$ and $x \otimes(u, v) \mapsto(x u, x v)$ respectively. Finally, when $B$ contains more than one split quadratic $F$-algebra, then it is completely split, and isomorphic to $F^{4}$; we see then $K, K^{\prime}, K^{\prime \prime}$ as fixed points of the involutions $(t, u, v, w) \mapsto(v, w, t, u),(w, v, u, t),(u, t, w, v)$ respectively.

In each case, we have $\eta_{K / F} \eta_{K^{\prime} / F} \eta_{K^{\prime \prime} / F}=1$. This implies that the product $\lambda_{K / F}(\psi) \lambda_{K^{\prime} / F}(\psi) \lambda_{K^{\prime \prime} / F}(\psi)$ is independent of the character $\psi$; we denote it by $\lambda_{B / F}$. If $\theta$ is a character of $B^{\times}$, we define for $\chi$ and $\psi$ as above

$$
\gamma_{\theta}^{F}(\chi, \psi)=\lambda_{B / F} \gamma^{B}\left(\theta \chi \circ N_{B / F}, \psi \circ T_{B / F}\right) .
$$

For the number $L_{B / F}$ defined in 1.2 , we have

$$
L_{B / F}=L_{K / F} L_{K^{\prime} / F} L_{K^{\prime \prime} / F},
$$

expressing the inductivity property of the $L$-function. The discriminant $D_{B / F}$ satisfies a similar relation ([S, Chapter VI.2] with the Artin representation, and [W2, Corollary 2 of Theorem 5, Chapter XII.4] with the Herbrand distribution):

$$
D_{B / F}=D_{K / F} D_{K^{\prime} / F} D_{K^{\prime \prime} / F} \text {. }
$$

Also, we have the relations

$$
\eta_{B / K}=\eta_{K^{\prime} / F} \circ N_{K / F}
$$

In particular, the elements of $F$ and those of $K_{1}=\operatorname{Ker} N_{K / F}$ are norms irom elements of $B$. We remark also that the conjugation of $B$ over $K^{\prime}$ when restricted to $K$ induces the conjugation of $K$ over $F$. Finally, the product $\lambda_{B / F} \eta_{K / F}(-1)$ is the factor $\lambda_{B / K}\left(\psi \circ T_{K / F}\right)$ for any $\psi$ as above. We write it simply $\lambda_{B / K}$.

\section{Preliminary Results}

2.1. Some measures. Let $K$ be a degree $n$ étale $F$-algebra, with its trace form $T$ and its norm form $N$. The bilinear form $(x, y) \mapsto T(x y)$ on $K$ is 
nondegenerate; hence, it defines a self-pairing on the top exterior power $\wedge^{n} K$, and a Haar measure $d_{K / F}$ on $K$. In other words, we have, by definition of the discriminant $D_{K / F}$,

$$
\int_{\mathscr{O}_{K}} d_{K / F} x=D_{K / F}^{-1 / 2}
$$

The maps $x \mapsto x / T x$ and $x \mapsto T x$ give a decomposition of the complement in $K$ of the hyperplane $\operatorname{Ker} T$ as the product of the affine hyperplane $K_{T}$ consisting of trace 1 elements, by $F^{\times}$. This in turn yields a decomposition of the differential forms on $K$, hence a decomposition of the Haar measure $d_{K / F} x$ as $|T x|^{n-1} d_{T}(x / T x) d T x$, where $d_{T} y$ is a measure on $K_{T}$ invariant under translations by $\operatorname{Ker} T$. This is also

$$
\frac{d_{K / F} x}{|N x|}=\frac{d_{T}(x / T x)}{|N(x / T x)|} \frac{d T x}{|T x|} .
$$

When passed to the normalized Haar measures on $K^{\times}$and $F^{\times}$, this defines a measure $d^{\bullet} y$ on $K_{T}$ by

$$
d_{K}^{\times} x=d^{\bullet}(x / T x) d_{F}^{\times} T x .
$$

In terms of the measure $d_{T}$ introduced above and the number $L_{K}$ in (1.2) this gives

$$
d^{\bullet} y=D_{K / F}^{1 / 2} \frac{L_{K}}{L_{F}} \frac{d_{T} y}{|N y|} .
$$

When $K$ is a field, this can also be stated as follows. The projective space deduced from $K$ as an $n$-dimensional space over $F$ is also the factor group $K^{\times} / F^{\times}$; the affine hyperplane $K_{T}$ of $K$ imbeds in $K^{\times} / F^{\times}$as the open subset image by $x \mapsto x / T x$ of the complement of the hyperplane $\operatorname{Ker} T$ of $K$; the Haar measure on $K^{\times} / F^{\times}$given by the quotient of the normalized Haar measures on $K^{\times}$and $F^{\times}$induces on this open subset the measure $d^{\bullet} y$. It is a bounded measure since it gives to $K_{T}$ the volume of $K^{\times} / F^{\times}$with respect to the quotient measure $d_{K}^{\times} x / d_{F}^{\times} t$; this volume is computed by integrating the characteristic function of units in $K^{\times}$, which has volume 1: this gives

$$
\int_{K_{T}} d^{\bullet} y=\int_{K^{\times} / F^{\times}}\left(d^{\times} x / d^{\times} t\right)=\left[\left|K^{\times}\right|_{K}:\left|F^{\times}\right|_{K}\right]=e_{K / F}
$$

with $e_{K / F}$ the ramification index of $F$ in $K$.

If $K$ is a separable quadratic extension of $F$, with conjugation $x \mapsto \bar{x}$, then the homography $x \mapsto x^{-1}-1$ of the projective line $K \cup\{\infty\}$ sends $K_{T} \cup\{\infty\}$ onto $K_{1}$ since $N\left(x^{-1}-1\right)-1=(1-T x) / N x$. As $y^{-1}-1=\bar{y} / y$ for $y \in K_{T}$, this map coincides on $K_{T}$ with $x \mapsto \bar{x} / x$, which is a homomorphism from $K^{\times}$onto $K_{1}$ (by Hilbert Theorem 90) with kernel $F^{\times}$. Hence it identifies the groups $K_{1}$ and $K^{\times} / F^{\times}$in a compatible way with the two maps $K_{T} \rightarrow K^{\times} / F^{\times}$ 
and $K_{T} \rightarrow K_{1}$. This shows that, for $f$ an integrable function on $K_{T}$ with respect to the measure $d^{\bullet} y$,

$$
\int_{K_{T}} f(y) d^{\bullet} y=e_{K / F} \int_{K_{1}} f\left((1+w)^{-1}\right) d^{\times} w,
$$

where $d^{\times} w$ is the normalized Haar measure on $K_{1}$ since the ramification index $e_{K / F}$ is the volume of $K_{T}$ under $d^{\bullet} y$. If $K$ is $F \times F$, then $K_{1}$ is isomorphic to $F^{\times}$by $t \mapsto\left(t, t^{-1}\right)$, and $x \mapsto x^{-1}-1$ sends $(K \cup\{\infty\}) \backslash(\{0\} \times F) \cup(F \times\{0\})$ onto $F^{\times}$, and $\left(K_{T} \cup\{\infty\}\right) \backslash\{(1,0),(0,1)\}$ bijectively onto $F^{\times}$since $y^{-1}-1=$ $\bar{y} / y$ for $y \in K_{T}$. In this case, for $f$ an integrable function on $K_{T}$ with respect to $d^{\bullet} y=d y_{1} /\left|y_{1}\left(1-y_{1}\right)\right|$ at $y=\left(y_{1}, y_{2}\right)$, we have

$$
\int_{K_{T}} f(y) d^{\bullet} y=\int_{F^{\times}} f\left(\left(1+t^{-1}\right)^{-1},(1+t)^{-1}\right) d^{\times} t .
$$

\subsection{A formula for the gamma functions $\Gamma^{K}$.}

Lemma. Let $K$ be a finite separable extension of $F$, and let $K_{T}$ be the affine $F$-hyperplane of $K$ consisting of elements $y$ with trace $T y=1$. If $\mu \in \mathscr{A}\left(K^{\times}\right)$ has the module of its restriction to $F^{\times}$larger than $q^{-1}$, then $\mu$ is integrable on $K_{T}$ with respect to the measure $d^{\bullet} y$ and

$$
\int_{K_{T}} \mu(y) d^{\bullet} y=\Gamma^{K}(\mu, \psi \circ T) / \Gamma\left(\left.\mu\right|_{F^{\times}}, \psi\right) .
$$

The proof proceeds by analytic continuation from the case $q_{K}^{-1 / n}<|\mu|<1$, where $n$ is the degree of $K$ over $F$; this later case is straightforward.

2.3. The pairing $\langle,\rangle_{\psi}$. Let $h(\chi, \psi)$ and $h^{\prime}(\chi, \psi)$ be two rational functions of the characters $\chi$ of $F^{\times}$, depending on the nontrivial additive character $\psi$ of $F$. We assume they satisfy the following conditions:

(a) there are numbers $a, a^{\prime}$ and $\omega, \omega^{\prime} \in \mathscr{A}\left(F^{\times}\right)$such that, for $\chi$ of large enough conductor,

$$
\begin{gathered}
h(\chi, \psi)=a \gamma^{F}(\chi, \psi) \gamma^{F}(\chi \omega, \psi), \\
h^{\prime}(\chi, \psi)=a^{\prime} \gamma^{F}(\chi, \psi) \gamma^{F}\left(\chi \omega^{\prime}, \psi\right) ;
\end{gathered}
$$

(b) no pole of $h$ is the inverse of a pole of $h^{\prime}$.

We define then $\left\langle h \mid h^{\prime}\right\rangle_{\psi} \in \mathbf{C} \cup\{\infty\}$ as follows. Observe that for $\mu \in \mathscr{A}\left(F^{\times}\right)$, the function $\chi \mapsto h(\chi \mu, \psi)$ satisfies a) with $\omega$ replaced by $\omega \mu^{2}$; except for a finite number of $\mu$ 's, condition $\mathrm{b})$ is satisfied for $h(\chi \mu, \psi)$ and $h^{\prime}(\chi, \psi)$. We denote by $d \chi$ the 1 -form on $\mathscr{A}\left(F^{\times}\right)$read as $\frac{1}{2 \pi i} Z^{-1} d Z$ on each connected component $\chi \mathbf{C}^{\times}$. Due to property (a), for $n$ large enough, say, $n>N$, the integral

$$
\oint_{a(\chi)=n} h(\chi Z, \psi) h^{\prime}\left(\chi^{-1}, \psi\right) d \chi, \quad Z \in \mathbf{C}^{\times},
$$


taken on simple positive contours around the origin in each component of conductor $a(\chi)=n$, is 0 if $\omega \omega^{\prime}$ ramifies, and otherwise is

$$
\begin{aligned}
& a a^{\prime} \omega(-1) \oint_{a(\chi)=n} Z^{-2 \operatorname{ord} \psi-2 n}\left(\omega \omega^{\prime}\right)^{-\operatorname{ord} \psi-n} d \chi \\
& \quad=a a^{\prime}\left(1-q^{-1}\right)^{2} Z^{-2 \operatorname{ord} \psi}\left(\omega \omega^{\prime}\right)^{-\operatorname{ord} \psi}\left(q Z^{-2}\left(\omega \omega^{\prime}\right)^{-1}\right)^{n} ;
\end{aligned}
$$

hence for $\left|Z^{2} \omega \omega^{\prime}\right|>q$, the series

$$
\sum_{n>N} \oint_{a(\chi)=n} h(\chi Z, \psi) h^{\prime}\left(\chi^{-1}, \psi\right) d \chi
$$

converges absolutely with sum

$$
a a^{\prime} \omega(-1)\left(1-q^{-1}\right)^{2}\left(Z^{2} \omega \omega^{\prime}\right)^{-\operatorname{ord} \psi} \frac{\left(Z^{2} \omega \omega^{\prime}\right)^{-N-1} q^{N+1}}{1-q Z^{-2}\left(\omega \omega^{\prime}\right)^{-1}} .
$$

Then we define the number $\left\langle h \mid h^{\prime}\right\rangle_{\psi}$ as the finite part of the integral

$$
\oint_{\mathscr{A}\left(F^{\times}\right)} h(\chi, \psi) h^{\prime}\left(\chi^{-1}, \psi\right) d \chi,
$$

where a simple positive contour around the origin is taken in each component of $\mathscr{A}\left(F^{\times}\right)$, containing the poles of $\chi \mapsto h^{\prime}\left(\chi^{-1}, \psi\right)$ but not those of $\chi \mapsto$ $h(\chi, \psi)$. This means that $\left\langle h \mid h^{\prime}\right\rangle_{\psi}$ is given for $N$ large enough by

$$
\begin{aligned}
\left\langle h \mid h^{\prime}\right\rangle_{\psi}= & \oint_{a(\chi) \leq N} h(\chi, \psi) h^{\prime}\left(\chi^{-1}, \psi\right) d \chi \\
& +\left\{\begin{array}{l}
0 \quad \text { if } \omega \omega^{\prime} \text { ramifies, } \\
a a^{\prime} \omega(-1)\left(1-q^{-1}\right)^{2} \frac{\left(\omega \omega^{\prime}\right)^{-N-\text { ord } \psi}}{\omega \omega^{\prime}-q}-q^{N+1} \text { otherwise. }
\end{array}\right.
\end{aligned}
$$

It is finite unless $\omega \omega^{\prime}=q$. In the case $h^{\prime}\left(\chi^{-1}, \psi\right)=b h(\chi, \psi)^{-1}$, we have $a a^{\prime} \omega(-1)=b, \omega^{\prime}=\omega^{-1}$, so $\omega \omega^{\prime}$ is not $q$ and

$$
\left\langle h \mid h^{\prime}\right\rangle_{\psi}=b \oint_{a(\chi) \leq N} d \chi+b\left(1-q^{-1}\right)^{2} \frac{q^{N+1}}{1-q} ;
$$

since $\oint_{a(\chi) \leq N} d \chi$ is the measure of units $t$ satisfying $\operatorname{ord}(t-1) \geq N$, that is,

$$
q^{N},\left(1-q^{-1}\right)=-\left(1-q^{-1}\right)^{2} \frac{q^{N+1}}{1-q},
$$

we have shown that $\left\langle h \mid h^{\prime}\right\rangle_{\psi}=0$ in this case.

We shall write

$$
\left\langle h \mid h^{\prime}\right\rangle_{\psi}=\oint_{\mathscr{A}\left(F^{\times}\right)} h(\chi, \psi) h^{\prime}\left(\chi^{-1}, \psi\right) d \chi .
$$

An example of functions $h, h^{\prime}$ satisfying (a) and (b) are the quadratic Gauss sums $\gamma_{\theta}^{F}$ and $\gamma_{\theta^{\prime}}^{F}$. 
2.4. We give an example of the pairing involving the beta function. In general, for $\mu, \nu$ characters of $K^{\times}$, and $\psi$ a nontrivial additive character of $K$, we define the beta function $B^{K}$ of $K$ by

$$
B^{K}(\mu, \nu)=\Gamma^{K}(\mu, \psi) \Gamma^{K}(\nu, \psi) / \Gamma^{K}(\mu \nu, \psi),
$$

which is independent of the choice of $\psi$. We have used the traditional notation $B^{K}$.

Proposition. Let $K$ be a separable quadratic extension of $F$, and $\theta, \theta^{\prime} \in$ $\mathscr{A}\left(K^{\times}\right)$. Assume $\left|\theta \theta^{\prime}\right|>q_{K}^{-1 / 2}$, and $\theta \theta^{\prime} \neq 1$ if $\theta$ and $\theta^{\prime}$ are liftings of characters of $F^{\times}$. Then

$$
\int_{\mathscr{A}\left(F^{\times}\right)} B^{K}\left(\theta \chi \circ N, \theta^{\prime} \chi^{-1} \circ N\right) d \chi=\int_{K_{T}}\left(\theta \bar{\theta}^{\prime}\right)(y) d^{\bullet} y,
$$

where the left-hand side is defined from the pairing in $\S 2.3$, and $d^{\bullet} y$ has been defined in $\S 2.1$.

Proof. The condition $\left|\theta \theta^{\prime}\right|>q_{K}^{-1 / 2}$ assures the convergence of both integrals. As they are analytic in this domain, we prove the identity under the conditions $|\theta|<1,\left|\theta^{\prime}\right|<1,\left|\theta \theta^{\prime}\right|>q_{K}^{-1 / 2}$. Let $m$ be a positive integer and choose a positive number $R_{m}$ satisfying

$$
\Gamma^{K}(\theta \chi \circ N, \psi \circ T)=\int_{|N x| \leq R_{m}} \theta(x) \chi(N x) \psi(T x) d^{\times} x,
$$

and

$$
\Gamma^{K}\left(\theta^{\prime} \chi^{-1} \circ N, \psi \circ T\right)=\int_{|N y| \leq R_{m}} \theta^{\prime}(y) \chi^{-1}(N y) \psi(T y) d^{\times} y
$$

for all characters $\chi \in \mathscr{A}\left(F^{\times}\right)$with conductor $a(\chi) \leq m$. The orthogonal in $F^{\times}$of this subgroup of $\mathscr{A}\left(F^{\times}\right)$is $1+\mathscr{P}^{m}$, where $\mathscr{P}$ is the valuation ideal of $F$. So $\Gamma^{K}\left(\theta \theta^{\prime}, \psi \circ T\right)$ times the left-hand side of (2.4.2) is the limit as $m$ tends to infinity of

$$
\oint_{a(\chi) \leq m}\left(\int_{|N x| \leq R_{m},|N y| \leq R_{m}} \theta(x) \theta^{\prime}(y) \psi(T x+T y) \chi(N x / N y) d^{\times} x d^{\times} y\right) d \chi,
$$

which is equal to

$$
\begin{aligned}
\left|\mathscr{O}^{\times} /\left(1+\mathscr{P}^{m}\right)\right| \int_{|N x| \leq R_{m},|N y| \leq R_{m}, N(y / x) \in 1+\mathscr{P}^{m}} \theta(x) \theta^{\prime}(y) \psi(T x+T y) d^{\times} x d^{\times} y \\
=\left|\mathscr{O}^{\times} /\left(1+\mathscr{P}^{m}\right)\right| \int_{N w \in 1+\mathscr{P}^{m},|N x| \leq R_{m}|N(1+w)|}\left(\theta \theta^{\prime}\right)(x) \psi(T x) \theta^{\prime}(w) \\
\times\left(\theta \theta^{\prime}\right)(1+w)^{-1} d^{\times} x d^{\times} w,
\end{aligned}
$$

by the change of variables $(x, y) \mapsto\left(x(1+w)^{-1}, x w(1+w)^{-1}\right)$. For $m$ large enough, the subgroup $1+\mathscr{P}^{m}$ is the image by $N$ of some subgroup $1+\mathscr{P}_{K}^{m^{\prime}}$ such that $w \mapsto \theta^{\prime}(w)\left(\theta \theta^{\prime}\right)(1+w)^{-1}$ on $N^{-1}\left(1+\mathscr{P}^{m}\right)=K_{1}\left(1+\mathscr{P}_{K}^{m^{\prime}}\right)$ is constant 
$\bmod \left(1+\mathscr{P}_{K}^{m^{\prime}}\right)$; then, because $\left|\mathscr{O}^{\times} /\left(1+\mathscr{P}^{m}\right)\right| \int_{1+\mathscr{P}^{m}} d^{\times} t=1$, our expression is, with the normalized Haar measure $d^{\times} w$ on $K_{1}$,

$$
e \int_{x \in K^{\times}, w \in K_{1},|N x| \leq R_{m}|N(1+w)|}\left(\theta \theta^{\prime}\right)(x) \psi(T x) \theta^{\prime}(w)\left(\theta \theta^{\prime}\right)(1+w)^{-1} d^{\times} x d^{\times} w .
$$

We choose now a positive number $r$ for which

$$
\Gamma^{K}\left(\theta \theta^{\prime}, \psi \circ T\right)=\int_{|N x| \leq r}\left(\theta \theta^{\prime}\right)(x) \psi(T x) d^{\times} x .
$$

Then, for $|N(1+w)|>r / R_{m}$, the ball $|N x| \leq R_{m}|N(1+w)|$ contains the ball $|N x| \leq r$. We write our expression as

$$
\begin{aligned}
& e \Gamma^{K}\left(\theta \theta^{\prime}, \psi \circ T\right) \int_{w \in K_{1},|N(1+w)|>r / R_{m}} \theta^{\prime}(w)\left(\theta \theta^{\prime}\right)(1+w)^{-1} d^{\times} w \\
& +e \int_{\substack{x \in K^{\times}, w \in K_{1} \\
|N x| \leq R_{m}|N(1+w)|,|N(1+w)| \leq r / R_{m}}}\left(\theta \theta^{\prime}\right)(x) \psi(T x) \theta^{\prime}(w) \\
& \times\left(\theta \theta^{\prime}\right)(1+w)^{-1} d^{\times} x d^{\times} w .
\end{aligned}
$$

By Cayley transform $y=(1+w)^{-1}, \theta^{\prime}(w)\left(\theta \theta^{\prime}\right)(1+w)$ becomes $\left(\theta \bar{\theta}^{\prime}\right)(y)=$ $\theta(y) \theta^{\prime}(\bar{y})$, and the first term is

$$
\Gamma^{K}\left(\theta \theta^{\prime}, \psi \circ T\right) \int_{K_{T},|N y|<R_{m} / r}\left(\theta \bar{\theta}^{\prime}\right)(y) d^{\bullet} y .
$$

The assumption $\left|\theta \theta^{\prime}\right|>q_{K}^{-1 / 2}$ implies that this integral has a limit when $m$, hence $R_{m}$, goes to infinity, by Lemma 2.2. We prove now that the second term goes to 0 . Let $\sigma$ be the real number such that $\left|\theta \theta^{\prime}(x)\right|=|N x|^{\sigma}$. By assumption, $\sigma<1 / 2$. The second term now reads

$$
\int_{\substack{x \in K^{\times}, y \in K_{T} \\|N(x y)| \leq R_{m},|N y| \geq R_{m} / r}}\left(\theta \theta^{\prime}\right)(x)\left(\theta \bar{\theta}^{\prime}\right)(y) \psi(T x) d^{\times} x d^{\bullet} y
$$

and, in absolute value, is dominated by

$$
\begin{aligned}
& \int_{\substack{x \in K^{\times}, y \in K_{T} \\
|N(x y)| \leq R_{m},|N y| \geq R_{m} / r}}|N(x y)|^{\sigma} d^{\times} x d^{\bullet} y \\
& =\int_{x \in K^{\times},|N x| \leq R_{m}}|N x|^{\sigma} d^{\times} x \int_{y \in K_{T},|N y| \geq R_{m} / r} d^{\bullet} y .
\end{aligned}
$$

In the right-hand side, the first integral is $O\left(R_{m}^{\sigma}\right)$, the second is $O\left(R_{m}^{-1 / 2}\right)$ as seen in the proof of lemma. So, the second term is $O\left(R_{m}^{\sigma-1 / 2}\right)$, and goes to 0 when $m$ goes to infinity. This achieves the proof of the proposition.

2.5. Given two quadratic étale $F$-algebras $K$ and $K^{\prime}$, and $B=K \otimes_{F} K^{\prime}$, we denote by $B_{*}$ the subgroup of $K^{\times} \times K^{\prime \times}$ consisting of elements $\left(x, x^{\prime}\right)$ such 
that $N_{K / F} x=N_{K^{\prime} / F^{\prime}} x^{\prime}$. It is a closed subgroup of $K^{\times} \times K^{\prime \times}$ and we have a homomorphism from $B^{\times}$to $B_{*}$ given by

$$
x \mapsto\left(N_{B / K} x, N_{B / K^{\prime}} x\right)
$$

with kernel $K_{1}^{\prime \prime}=\operatorname{Ker} N_{K^{\prime \prime} / F}$, where $K^{\prime \prime}$ denotes the third quadratic sub- $F$ algebra in $B$. As $\mathscr{O}_{K}^{\times} \times \mathscr{O}_{K^{\prime}}^{\times}$is the maximal compact subgroup of $K^{\times} \times K^{\prime \times}$, its intersection $\mathscr{O}_{B *}^{\times}$with $B_{*}$ is the maximal compact subgroup of $B_{*}$, and it is open in $B_{*}$. The Haar measure $d_{B_{*}}^{\times}$gives to $\mathscr{O}_{B_{*}}^{\times}$the volume 1 . The group $B_{*}$ appears also as the orthogonal in $K^{\times} \times K^{\prime \times}$ of the group $\mathscr{A}\left(F^{\times}\right)$embedded in $\mathscr{A}\left(K^{\times} \times K^{\prime \times}\right)$ by $\chi \mapsto\left(\chi \circ N_{K / F}, \chi^{-1} \circ N_{K^{\prime} / F}\right)$. By Poisson summation formula [W1], this implies that there is a number $c_{1}>0$ such that, for $h$ in the Schwartz-Bruhat space $\mathscr{S}\left(K^{\times} \times K^{\prime \times}\right)$ one has

$$
\begin{aligned}
& \int_{\mathscr{A}\left(F^{\times}\right)}\left(\int_{K^{\times} \times K^{\prime \times}} h\left(x, x^{\prime}\right) \chi\left(N_{K / F} x / N_{K^{\prime} / F^{\prime}} x^{\prime}\right) d_{K}^{\times} x d_{K^{\prime}}^{\times} x^{\prime}\right) d \chi \\
& =c_{1} \int_{B^{*}} h\left(x, x^{\prime}\right) d_{B_{*}}^{\times}\left(x, x^{\prime}\right) .
\end{aligned}
$$

Lemma. Assume that $K$ and $K^{\prime}$ are not isomorphic. Then

(a) the image of $B^{\times}$in $B_{*}$ by (2.5.1) is an index 2 subgroup;

(b) the restriction of $(2.5 .1)$ to $\mathscr{O}_{B}^{\times}$has image in $\mathscr{O}_{B_{*}}^{\times}$a subgroup of index $e_{K^{\prime \prime} / F}$ if $K$ or $K^{\prime}$ splits over $F$, and of index 2 otherwise;

(c) $c_{1}=1$ if $K$ or $K^{\prime}$ splits over $F$, otherwise $c_{1}=f_{K^{\prime \prime} / F}$, the modular degree of $K^{\prime \prime}$ over $F$.

Proof. (1) Assume first $K^{\prime}=F \times F$. Then $B_{*}$ is the set of $(w,(u, v)) \in$ $K^{\times} \times(F \times F)^{\times}$such that $w \bar{w}=u v$. The algebra $B$ is $K \times K$ and (2.5.1) is $(x, y) \mapsto(x y,(x \bar{x}, y \bar{y}))$. We check now that the image of $B^{\times}$is the kernel of the map $(w,(u, v)) \mapsto \eta_{K / F}(u)$ on $B_{*}$ : if $(w,(u, v))$ lies in $B_{*}$ and $u$ is a norm $x \bar{x}$ from $K^{\times}$, so is $v=w \bar{w} / u$. Put $y=w x^{-1}$, then $v=y \bar{y}$ and $w=x y$. Thus $(w,(u, v))=(x y,(x \bar{x}, y \bar{y}))$ lies in the image of $B^{\times}$. This gives an isomorphism between the cokernel of the map (2.5.1) and the cokernel of $\eta_{K / F}$, and proves (a) in our case. For (b), we observe that the inverse image of $\mathscr{O}_{B_{*}}^{\times}$in $B^{\times}$is the group $\mathscr{O}_{B}^{\times}$of units of $B$, and that the cokernel of (2.5.1) restricted to $\mathscr{O}_{B}^{\times}$is isomorphic to $\mathscr{O}_{F}^{\times} / N_{K / F} \mathscr{O}_{K}^{\times}$, which has order equal to the ramification index $e_{K / F}$ of $K$ over $F$. For (c), we apply (2.5.2) to the characteristic function of $\mathscr{O}_{K}^{\times} \times \mathscr{O}_{K^{\prime}}^{\times}:$the integral

$$
\int_{\mathscr{O}_{K}^{\times} \times \mathscr{G}_{F}^{\times} \times \mathscr{G}_{F}^{\times}} \chi(w \bar{w}) \chi^{-1}(u v) d_{K}^{\times} w d_{F}^{\times} u d_{F}^{\times} v
$$

is 0 unless $\chi$ is unramified, and then the left-hand side of (2.5.2) is 1 , as is the right-hand side when $c_{1}=1$. This proves the lemma when $K$ or $K^{\prime}$ splits. 
(2) Assume now that $K$ and $K^{\prime}$ are not isomorphic and nonsplit. Let $(u, v) \in B_{*}$; then the equality $N_{K / F} u=N_{K^{\prime} / F} v$ shows that this element of $F^{\times}$lies in $\operatorname{Im} N_{K / F} \cap \operatorname{Im} N_{K^{\prime} / F}=\operatorname{Im} N_{B / F}$. Let $z \in B^{\times}$with $N_{B / F} z$ equal to this common norm. Its image by $(2.5 .1)$ differs from $(u, v)$ by some element of $K_{1} \times K_{1}^{\prime}$, the product of $\operatorname{Ker} N_{K / F}$ by $\operatorname{Ker} N_{K^{\prime} / F}$. As the inverse image of $K_{1} \times K_{1}^{\prime}$ in $B^{\times}$by (2.5.1) is $B_{1}=\operatorname{Ker} N_{B / F}$, we have shown that the orbits of $B^{\times}$acting on $B_{*}$ through (2.5.1) are the same as the orbits of $B_{1}$ acting on $K_{1} \times K_{1}^{\prime}$. As any element of $K_{1}$ lies in $\operatorname{Im} N_{B / K}$, so is in $N_{B / K} B_{1}$, the number of these orbits is $\left[K_{1}^{\prime}: N_{B / K^{\prime}} B_{K}\right]$ where $B_{K}=\operatorname{Ker} N_{B / K}$. We prove now that $N_{B / K^{\prime}} B_{K}$ has index 2 in $K_{1}^{\prime}$. For that, we use the long exact sequence for the cohomology of the group $\mathrm{Gal} B / K^{\prime \prime}$ acting on the exact sequence

$$
1 \rightarrow K^{\times} \rightarrow B^{\times} \rightarrow B_{K} \rightarrow 1
$$

of subgroups of $B^{\times}$by its Galois action composed with inversion:

$$
\begin{aligned}
1 \rightarrow K_{1} \rightarrow B_{K^{\prime \prime}} & \rightarrow K_{1}^{\prime} \rightarrow F^{\times} / N_{K / F} K^{\times} \stackrel{\alpha}{\rightarrow} K^{\prime \prime \times} / N_{B / K^{\prime \prime}} B^{\times} \\
\rightarrow & K_{1}^{\prime \prime} / N_{B / K^{\prime \prime}} K^{\times} \rightarrow 0 \rightarrow \cdots .
\end{aligned}
$$

As any element of $F^{\times}$is a norm from $B$ to $K^{\prime \prime}$, the arrow $\alpha$ is 0 . The image of $B_{K^{\prime \prime}}$ in $K_{1}^{\prime}$ is $N_{B / K^{\prime}} B_{K}$, and the factor group is isomorphic to $F^{\times} / N_{K / F} K^{\times}$, which has order 2. This proves the claim, which gives part (a) of the lemma and also part (b) since $B_{1}$ consists of units. For (c), we use again the characteristic function of $\mathscr{O}_{K}^{\times} \times \mathscr{O}_{K^{\prime}}^{\times}$; this shows that $c_{1}$ is the index in $\mathscr{O}_{F}^{\times}$of the subgroup generated by $N_{K / F} \mathscr{O}_{F}^{\times}$and $N_{K^{\prime} / F} \mathscr{O}_{K^{\prime}}^{\times}$. As $N_{K / F} \mathscr{O}_{K}^{\times}=\mathscr{O}_{F}^{\times}$if $K$ is unramified over $F$, and $\left[\mathscr{O}_{F}^{\times}: N_{K / F} \mathscr{O}_{K}^{\times}\right]=2$ otherwise, we see that $c_{1}=1$ unless $N_{K / F} \mathscr{O}_{K}^{\times}=N_{K^{\prime} / F} \mathscr{O}_{K^{\prime}}^{\times}$; in this case, the units of $F$ are either in $N_{K / F} K^{\times} \cap N_{K^{\prime} / F} K^{\prime \times}$ or in the complement of $N_{K / F} K^{\times} \cup N_{K^{\prime} / F} K^{\prime \times}$, that is, are all in $N_{K^{\prime \prime} / F} K^{\prime \prime \times}$ since $F^{\times}$is the union $\operatorname{Im} N_{K / F} \cup \operatorname{Im} N_{K^{\prime} / F} \cup \operatorname{Im} N_{K^{\prime \prime} / F}$; but then, $K^{\prime \prime}$ is unramified, so $f_{K^{\prime \prime} / F}=2$. This gives the proof of (c).

\section{Main Results}

3.1. Theorem 1. Let $K$ and $K^{\prime}$ be two quadratic étale $F$-algebras, and let $B$ be their composite algebra $K \otimes_{F} K^{\prime}$. Let $\theta, \theta^{\prime}$ be characters of $K^{\times}$and $K^{\prime \times}$ respectively. Denote by $\omega \eta_{K / F}, \omega^{\prime} \eta_{K^{\prime} / F}$ the restrictions of $\theta, \theta^{\prime}$ to $F^{\times}$, and by $\theta \times \theta^{\prime}$ the character $\left(\theta \circ N_{B / K}\right)\left(\theta^{\prime} \circ N_{B / K^{\prime}}\right)$ of $B^{\times}$. Then we have

$$
\left\langle\gamma_{\theta}^{F} \mid \gamma_{\theta^{\prime}}^{F}\right\rangle_{\psi}=\gamma_{\theta \times \theta^{\prime}}^{F}\left(q^{-1 / 2}, \psi\right) / \Gamma\left(\omega \omega^{\prime} q^{-2}, \psi\right) .
$$

Proof. (a) If $K$ and $K^{\prime}$ are both split, we see them in $B=F^{4}$ as the fixed points of the involutions

$$
(t, u, v, w) \mapsto(v, w, t, u)
$$


and

$$
(t, u, v, w) \mapsto(w, v, u, t)
$$

respectively. For $\theta=\mu \otimes \nu, \theta^{\prime}=\mu^{\prime} \otimes \nu^{\prime}$, we have

$$
\theta \times \theta^{\prime}=\left(\mu \mu^{\prime}\right) \otimes\left(\nu \nu^{\prime}\right) \otimes\left(\mu \nu^{\prime}\right) \otimes\left(\nu \mu^{\prime}\right) .
$$

In this case, formula (3.1.1) has been proved in [L and GL1].

(b) If $K$ and $K^{\prime}$ are isomorphic fields, we see them as fixed points in $B=$ $K \times K$ under the involutions $(x, y) \mapsto(y, x)$ and $(x, y) \mapsto(\bar{y}, \bar{x})$ respectively, with ${ }^{-}$the conjugation of $K$ over $F$. Then, $\theta \times \theta^{\prime}$ is the character $(x, y) \mapsto$ $\left(\theta \theta^{\prime}\right)(x)\left(\theta \bar{\theta}^{\prime}\right)(y)$, and

$$
\gamma_{\theta \times \theta^{\prime}}^{F}\left(q^{-1 / 2}, \psi\right)=\lambda_{B / K}(\psi)^{2} \gamma^{K}\left(\theta \theta^{\prime} q_{K}^{-1 / 2}, \psi \circ T\right) \gamma^{K}\left(\theta \bar{\theta}^{\prime} q_{K}^{-1 / 2}, \psi \circ T\right)
$$

with $T=T_{K / F}$. Multiply both $\theta$ and $\theta^{\prime}$ by $q_{K}^{1 / 2}$, then $\omega \omega^{\prime}$ is multiplied by $q^{2}$ and (3.1.1) takes the form

$$
\oint_{\mathscr{A}\left(F^{\times}\right)} B^{K}\left(\theta \chi \circ N, \theta^{\prime} \chi^{-1} \circ N\right) d \chi=\Gamma^{K}\left(\theta \bar{\theta}^{\prime}, \psi \circ T\right) / \Gamma\left(\omega \omega^{\prime}, \psi\right),
$$

with $N=N_{K / F}$ and $B^{K}$ the beta function (2.4.1) for $K$. Formula (3.1.2) is the proposition of $\S 2.4$ combined with the lemma of $\S 2.2$.

(c) The case of $K$ and $K^{\prime}$ nonisomorphic remains. As they play the same role in the statement, we assume that $K$ is a field. Then the third quadratic étale $F$-algebra $K^{\prime \prime}$ in $B$ defined by $K$ and $K^{\prime}$ is also a field, satisfying

$$
\eta_{K / F} \eta_{K^{\prime} / F} \eta_{K^{\prime \prime} / F}=1
$$

We choose two coset representatives, say, $a_{+}$and $a_{-}$of $N_{K^{\prime \prime} / F}\left(K^{\prime \prime \times}\right)$ in $F^{\times}$. Using the complement formula in $\S 1.1$ for $\Gamma$, we rewrite $(3.1 .1)$ as

$$
\left\langle\gamma_{\theta}^{F} \mid \gamma_{\theta^{\prime}}^{F}\right\rangle_{\psi}=|\psi|\left(\omega \omega^{\prime}\right)(-1) L_{F}^{-2} \Gamma\left(\omega^{-1} \omega^{\prime-1} q, \psi^{-1}\right) \gamma_{\theta \times \theta^{\prime}}^{F}\left(q^{-1 / 2}, \psi\right) .
$$

Since both sides of (3.1.3) are meromorphic functions in $\theta, \theta^{\prime}$, it suffices to prove the identity for $q<\left|\omega \omega^{\prime}\right|<q^{2}$, and we shall so assume. Our strategy is to express the right-hand side of (3.1.3) as an integral over the subgroup $B_{*}$ of $K^{\times} \times K^{\prime \times}$, the orthogonal of the group $\mathscr{A}\left(F^{\times}\right)$embedded in $\mathscr{A}\left(K^{\times} \times K^{\prime \times}\right)$ by $\chi \mapsto\left(\chi \circ N_{K / F}, \chi^{-1} \circ N_{K^{\prime} / F}\right)$; a suitable form of Poisson summation formula expresses then the right-hand side as a contour integral over $\mathscr{A}\left(F^{\times}\right)$, which is the left-hand side of (3.1.3).

The condition $\left|\omega \omega^{\prime}\right|>q$ implies the integrability near 0 for $d^{\times} t$ of the character $\omega^{-1} \omega^{\prime-1} q$. Thus, for $R$ large, one has

$$
\Gamma\left(\omega^{-1} \omega^{\prime-1} q, \psi^{-1}\right)=\int_{|t| \leq R}\left(\omega \omega^{\prime}\right)(t)^{-1} \psi(-t)|t|^{-1} d^{\times} t,
$$

and

$$
\Gamma\left(\omega^{-1} \omega^{-1} \eta^{\prime \prime} q, \psi^{-1}\right)=\int_{|t| \leq R}\left(\omega \omega^{\prime}\right)(t)^{-1} \eta^{\prime \prime}(t) \psi(-t)(t)^{-1} d^{\times} t
$$


with $\eta^{\prime \prime}=\eta_{K^{\prime \prime} / F}$, so

$$
\Gamma\left(\omega^{-1} \omega^{\prime-1} q, \psi^{-1}\right)=\Gamma_{+}+\Gamma_{-},
$$

where $\Gamma_{ \pm}$correspond to the intersection over those $t$ in the ball $|t| \leq R$ which satisfy $t \in a_{ \pm} N^{\prime \prime}\left(K^{\prime \prime \times}\right)$, with $N^{\prime \prime}=N_{K^{\prime \prime} / F}$. We express $\Gamma_{ \pm}$as integrals over $K^{\prime \prime}$ :

$$
\begin{aligned}
\Gamma_{ \pm} & =\Gamma_{ \pm, R} \\
& =c^{-1}\left|a_{ \pm}\right|^{-1} \int_{\left|N^{\prime \prime} w\right| \leq R}\left(\omega \omega^{\prime}\right)\left(a_{ \pm} N^{\prime \prime} w\right)^{-1} \psi\left(-a_{ \pm} N^{\prime \prime} w\right)\left|N^{\prime \prime} w\right|^{-2} d_{K^{\prime \prime} / F} w,
\end{aligned}
$$

with $c$ being the measure of $K_{1}^{\prime \prime}=\operatorname{Ker} N^{\prime \prime}$ under $d_{K^{\prime \prime} / F} w / d^{\times} t$.

The condition $\left|\omega \omega^{\prime}\right|<q^{2}$ assures that the character $\beta=\theta \times \theta^{\prime}$ of $B^{\times}$is integrable near 0 with respect to the measure $d_{B, \psi}$; so, for $S$ large, we have

$$
\gamma_{\beta}^{B}\left(q_{B}^{-1 / 2}, \psi \circ T_{B / F}\right)=\int_{|z|_{B} \leq S} \beta(z) \psi \circ T_{B / F}(z) d_{B, \psi} z,
$$

where $|z|_{B}=\max (|N x|,|N y|)$ if $K^{\prime}$ is split and $z$ in $B$ correspond to $(x, y)$ in $K \times K$, and $N=N_{K / F}$.

Our first step is to express the product of the two gamma functions in the right-hand side of (3.1.3) as follows:

\section{Lemma.}

$$
\Gamma\left(\omega^{-1} \omega^{\prime-1} q, \psi\right) \gamma_{\beta}^{B}\left(q_{B}^{-1 / 2}, \psi \circ T_{B / F}\right)=|\psi|^{-1} \lambda^{\prime \prime}(\psi)^{-1} c^{-1} c^{\prime} \lim _{Q \rightarrow \infty} A_{Q}
$$

where $\lambda^{\prime \prime}(\psi)=\lambda_{K^{\prime \prime} / F}(\psi)$ and

$$
A_{Q}=\int_{\left(x, x^{\prime}\right) \in B_{*},|N x| \leq Q}(\theta \psi \circ T)(x)\left(\theta^{\prime} \psi \circ T^{\prime}\right)\left(x^{\prime}\right) d_{B_{*}, \psi}^{*}\left(x, x^{\prime}\right),
$$

with $T=T_{K / F}, T^{\prime}=T_{K^{\prime} / F}, c^{\prime}$ is the index of the image of $\mathscr{O}_{B}^{\times}$in $\mathscr{O}_{B_{*}}^{\times}$by (2.5.1) and $d_{B_{*}, \psi}^{*}\left(x, x^{\prime}\right)=|N x| d_{B_{*}, \psi}\left(x, x^{\prime}\right)$.

Proof of the lemma. Choose $S$ large as above, then

$$
\begin{aligned}
& c \Gamma\left(\omega^{-1} \omega^{\prime-1} q, \psi^{-1}\right) \gamma^{B}\left(\beta q_{B}^{-1 / 2}, \psi \circ T_{B / F}\right) \\
& =c\left(\Gamma_{+}+\Gamma_{-}\right) \gamma^{B}\left(\beta q_{B}^{-1 / 2}, \psi \circ T_{B / F}\right)
\end{aligned}
$$

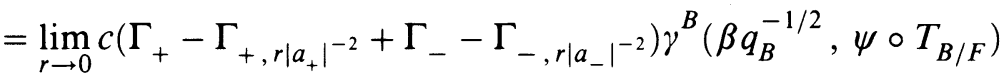

$$
\begin{aligned}
& =\lim _{r \rightarrow 0}\left(I\left(r, S, a_{+}\right)+I\left(r, S, a_{-}\right)\right),
\end{aligned}
$$

where, for $a=a_{+}$or $a_{-}$and $R$ large as before, we define

$$
\begin{aligned}
I(r, S, a)=|a|^{-1} \int_{|z|_{B} \leq S, r|a|^{-2} \leq\left|N^{\prime \prime} w\right| \leq R}\left(\omega \omega^{\prime}\right)\left(a N^{\prime \prime} w\right)^{-1} \psi\left(-a N^{\prime \prime} w\right) \beta(z) \\
\times \psi \circ T_{B / F}(z)\left|N^{\prime \prime} w\right|^{-2} d_{K^{\prime \prime} / F} w d_{B, \psi} z .
\end{aligned}
$$


Note that for $w \in K^{\prime \prime \times}$, we have

$$
\begin{aligned}
\beta(w) & =\theta\left(N_{B / K} w\right) \theta^{\prime}\left(N_{B / K^{\prime}} w\right)=\theta\left(N^{\prime \prime} w\right) \theta^{\prime}\left(N^{\prime \prime} w\right)=\left(\omega \omega^{\prime} \eta \eta^{\prime}\right)\left(N^{\prime \prime} w\right) \\
& =\left(\omega \omega^{\prime}\right)\left(N^{\prime \prime} w\right),
\end{aligned}
$$

with $\eta=\eta_{K / F}, \eta^{\prime}=\eta_{K^{\prime} / F}$ so that $\eta^{\prime \prime}=\eta \eta^{\prime}$. Therefore, $I(r, S, a)$ can be simplified as

$$
\begin{aligned}
&|a|^{-1}\left(\omega \omega^{\prime}\right)(a)^{-1} \int_{|z|_{B} \leq S, r|a|^{-2} \leq\left|N^{\prime \prime} w\right| \leq R} \beta\left(w^{-1} z\right) \psi\left(-a N^{\prime \prime} w\right) \\
& \times \psi \circ T_{B / F}(z)\left|N^{\prime \prime} w\right|^{-2} d_{K^{\prime \prime} / F} w d_{B, \psi} z \\
&=|a|^{-1}\left(\omega \omega^{\prime}\right)(a)^{-1} \int_{|z|_{B} \leq S r^{-1}|a|^{2}, r|a|^{-2} \leq\left|N^{\prime \prime} w\right| \leq R} \beta(z) \psi\left(-a N^{\prime \prime} w\right) \\
& \times \psi \circ T_{B / F}(w z) d_{K^{\prime \prime} / F} w d_{B, \psi} z
\end{aligned}
$$

by the change of variable $z \mapsto w z$. For a given $S$, the assumption $q<\left|\omega \omega^{\prime}\right|<$ $q^{2}$ implies that the integral

$$
\int_{|z|_{B} \leq S r^{-1},\left|N^{\prime \prime} w\right|<r}|\beta(z)| d_{K^{\prime \prime} / F} w d_{B, \psi} z
$$

is majorized by a constant multiple of $r \int_{|z|_{B} \leq S r^{-1}}|\beta(z)| d_{B} z$, which is $O\left(r^{\alpha}\right)$ with $\alpha=|\beta|^{\left(\log q_{B}\right)^{-1}}>0$. This shows that the difference

$$
I(r, S, a)-I\left(0, S r^{-1}|a|^{2}, a\right)
$$

tends to zero as $r$ does. Hence we have $c \Gamma\left(\omega^{-1} \omega^{\prime-1} q, \psi^{-1}\right) \gamma^{B}\left(\beta q_{B}^{-1 / 2}, \psi \circ T_{B / F}\right)=\lim _{Q \rightarrow \infty}\left(I\left(Q\left|a_{+}\right|^{2}, a_{+}\right)+I\left(Q\left|a_{-}\right|^{2}, a_{-}\right)\right)$ where for $R$ and $Q$ large $I(Q, a)=I(0, Q, a)$, that is,

$$
\begin{aligned}
I(Q, a)=|a|^{-1}\left(\omega \omega^{\prime}\right)(a)^{-1} \int_{|z|_{B} \leq Q,\left|N^{\prime \prime} w\right| \leq R} & \beta(z) \psi\left(-a N^{\prime \prime} w\right) \\
& \times \psi \circ T_{B / F}(w z) d_{K^{\prime \prime} F} w d_{B, \psi} z .
\end{aligned}
$$

For fixed $a$ and for $R$ large enough, the integral against $w$ has been computed in the lemma of $\S 1.5$. It is 0 unless $\left|N^{\prime \prime} \circ T_{B / K^{\prime \prime}} z\right| \leq R$; take $R$ large enough, the condition $|z|_{B} \leq Q$, or equivalently $\left|N_{B / K^{\prime \prime}} z\right|_{K^{\prime \prime}} \leq R$, implies $\left|T_{B / K^{\prime \prime}} z\right|_{K^{\prime \prime}} \leq R$, and this integral against $w$ is, for $|z|_{B} \leq Q$, equal to

$$
|\psi|^{-1}|a|^{-1} \eta^{\prime \prime}(a) \lambda^{\prime \prime}(\psi)^{-1} \psi\left(a^{-1} N^{\prime \prime} \circ T_{B / K^{\prime \prime}} z\right) \text {. }
$$

Noting

$$
\left(\omega \omega^{\prime}\right)(a)^{-1}=\theta(a)^{-1} \theta^{\prime}(a)^{-1} \eta(a) \eta^{\prime}(a)=\theta(a)^{-1} \theta^{\prime}(a)^{-1} \eta^{\prime \prime}(a)
$$

and

$$
N^{\prime \prime} \circ T_{B / K^{\prime \prime}}=T \circ N_{B / K}+T^{\prime} \circ N_{B / K^{\prime}}
$$


we arrive at

$$
\begin{aligned}
I\left(Q|a|^{2}, a\right)=|\psi|^{-1} \lambda^{\prime \prime}(\psi)^{-1}|a|^{-2} \int_{|z|_{B} \leq Q|a|^{2}} & (\theta \psi \circ T)\left(a^{-1} N_{B / K} z\right) \\
& \times\left(\theta \psi \circ T^{\prime}\right)\left(a^{-1} N_{B / K^{\prime}} z\right) d_{B / \psi^{\prime}} z .
\end{aligned}
$$

From the lemma in $\S 2.5$, the image of $B^{\times}$in $K^{\times} \times K^{\prime \times}$ by the map $\left(N_{B / K}\right.$, $\left.N_{B / K^{\prime}}\right)$ is a subgroup $B_{*}^{+}$of index 2 of $B_{*}$ with coset representatives $\left(a_{+}^{-1}, a_{+}^{-1}\right)$ and $\left(a_{-}^{-1}, a_{-}^{-1}\right)$. We express $I\left(Q|a|^{2}, a\right)$ as an integral over $B_{*}^{+}$:

$$
\begin{array}{r}
I\left(Q|a|^{2}, a\right)=|\psi|^{-1} \lambda^{\prime \prime}(\psi)^{-1} c^{\prime} \int_{\left(x, x^{\prime}\right) \in B_{*}^{+},|N x| \leq Q|a|^{2}}|a|^{-2}(\theta \psi \circ T)\left(a^{-1} x\right) \\
\times\left(\theta^{\prime} \psi \circ T^{\prime}\right)\left(a^{-1} x^{\prime}\right) d_{B_{*}, \psi}^{*}\left(x, x^{\prime}\right) \\
=|\psi|^{-1} \lambda^{\prime \prime}(\psi)^{-1} c^{\prime} \int_{\left(x, x^{\prime}\right) \in a^{-1} B_{*}^{+},|N x| \leq Q}(\theta \psi \circ T)(x)\left(\theta^{\prime} \psi \circ T^{\prime}\right)\left(x^{\prime}\right) d_{B_{*}, \psi}^{*}\left(x, x^{\prime}\right)
\end{array}
$$

by the change of variables $\left(x, x^{\prime}\right) \mapsto\left(a x, a x^{\prime}\right)$. Here $c^{\prime}$ is the index of the maximal compact subgroup of $B_{*}^{+}$in the maximal compact subgroup of $B_{*}$. Adding $I\left(Q\left|a_{+}\right|^{2}, a_{+}\right)$and $I\left(Q\left|a_{-}\right|^{2}, a_{-}\right)$leads to

$$
\begin{aligned}
& |\psi|^{-1} \lambda^{\prime \prime}(\psi)^{-1} c^{\prime} \int_{\left(x, x^{\prime}\right) \in B_{*},|N x| \leq Q}(\theta \psi \circ T)(x)\left(\theta^{\prime} \psi \circ T^{\prime}\right)\left(x^{\prime}\right) d_{B_{*}, \psi}^{*}\left(x, x^{\prime}\right) \\
& \quad=|\psi|^{-1} \lambda^{\prime \prime}(\psi)^{-1} A_{Q} .
\end{aligned}
$$

This proves the lemma.

Recall that $\lambda_{B / F}=\lambda(\psi) \lambda^{\prime}(\psi) \lambda^{\prime \prime}(\psi)$, where $\lambda(\psi)=\lambda_{K / F}(\psi), \lambda^{\prime}(\psi)=$ $\lambda_{K^{\prime} / F}(\psi), \lambda^{\prime \prime}(\psi)=\lambda_{K^{\prime \prime} / F}(\psi)$, as seen in $\S 1$. The lemma gives the right-hand side of (3.1.3) as

$(-i .5)$

$L_{F}^{-2} \lambda(\psi) \lambda^{\prime}(\psi) c^{-1} c^{\prime} \lim _{Q \rightarrow \infty} \int_{\left(x, x^{\prime}\right) \in B_{*},|N x| \leq Q}(\theta \psi \circ T)(x)\left(\theta^{\prime} \psi \circ T^{\prime}\right)\left(x^{\prime}\right) d_{B_{*}}^{*}\left(x, x^{\prime}\right)$.

On the other hand, for $\left|\omega \omega^{\prime}\right|<q^{2}$, the left-hand side of (3.1.3) is given by a convergent integral

$$
\lambda(\psi) \lambda^{\prime}(\psi) \oint_{\mathscr{A}\left(F^{\times}\right)} \gamma^{K}(\theta \chi \circ N, \psi \circ T) \gamma^{K^{\prime}}\left(\theta^{\prime} \chi^{-1} \circ N^{\prime}, \psi \circ T^{\prime}\right) d \chi .
$$

We assume now $|\omega|$ and $\left|\omega^{\prime}\right|<q$, which is compatible with the preceding assumption $q<\left|\omega \omega^{\prime}\right|<q^{2}$. For $\chi$ unitary with $a(\chi) \leq M$, the two gamma terms $\gamma^{K}(\theta \chi \circ N, \psi \circ T), \gamma^{K^{\prime}}\left(\theta^{\prime} \chi^{-1} \circ N^{\prime}, \psi \circ T^{\prime}\right)$ above are given by integrals on sufficiently large compact subsets of $K$ and $K^{\prime}$, depending on $M$, and independent of $\theta, \theta^{\prime}$ running through given compact subsets of $\mathscr{A}\left(K^{\times}\right)$, $\mathscr{A}\left(K^{\prime \times}\right)$ :

$$
\gamma^{K}(\theta \chi \circ N, \psi \circ T)=\int_{|N x| \leq Q(M)}(\theta \psi \circ T)(x) \chi \circ N(x) d_{K, \psi}^{*} x,
$$




$$
\gamma^{K^{\prime}}\left(\theta^{\prime} \chi^{-1} \circ N^{\prime}, \psi \circ T\right)=\int_{\left|x^{\prime}\right|_{K^{\prime}} \leq Q(M)}\left(\theta^{\prime} \psi \circ T^{\prime}\right)\left(x^{\prime}\right) \chi^{-1} \circ N^{\prime}\left(x^{\prime}\right) d_{K^{\prime}, \psi}^{*} x^{\prime} .
$$

Using (3.1.5), we see that identity (3.1.3) is equivalent to

$$
\left\{\begin{array}{l}
\lim _{M \rightarrow \infty} \chi_{a(\chi) \leq M}\left(\int_{|N x| \leq Q(M),\left|x^{\prime}\right|_{K^{\prime}} \leq Q(M)} f\left(x, x^{\prime}\right) x\left(N x / N^{\prime} x^{\prime}\right) d_{K, \psi}^{*} x d_{K^{\prime}, \psi^{\prime}}^{*} x^{\prime}\right) d \chi \\
=c^{-1} c^{\prime} L_{F}^{-2} \lim _{Q \rightarrow \infty} \int_{\left(x, x^{\prime}\right) \in B_{*},|N x| \leq Q} f\left(x, x^{\prime}\right) d_{B_{*}, \psi}^{*}\left(x, x^{\prime}\right),
\end{array}\right.
$$

where $f\left(x, x^{\prime}\right)=(\theta \psi \circ T)(x)\left(\theta^{\prime} \psi \circ T^{\prime}\right)\left(x^{\prime}\right)$ for $x \in K^{\times}, x^{\prime} \in K^{\prime \times}$. Recall that the measures $d_{K, \psi}^{*}, d_{K^{\prime}, \psi}^{*}, d_{B_{*}, \psi}^{*}$ are given by

$$
\begin{gathered}
d_{K, \psi}^{*} x=|\psi| D^{-1 / 2} L_{K}^{-1}|N x|^{1 / 2} d_{K}^{\times} x, \quad D=D_{K / F}, \\
d_{K^{\prime}, \psi}^{*} x^{\prime}=|\psi| D^{\prime-1 / 2} L_{K^{\prime}}^{-1}\left|N^{\prime} x^{\prime}\right|^{1 / 2} d_{K^{\prime}}^{\times} x^{\prime}, \quad D^{\prime}=D_{K^{\prime} / F}, \\
d_{B_{*}, \psi}^{*}\left(x, x^{\prime}\right)=|\psi|^{2} D_{B / F}^{-1 / 2} L_{B}^{-1}|N x|^{1 / 2}\left|N^{\prime} x^{\prime}\right|^{1 / 2} d_{B_{*}}^{\times}\left(x, x^{\prime}\right),
\end{gathered}
$$

hence, by $\S 1.6$, we rewrite $(3.1 .6)$ as

$$
\left\{\begin{array}{l}
\lim _{Q \rightarrow \infty} \chi_{a(\chi) \leq M}\left(\int_{|N x| \leq Q(M),\left|x^{\prime}\right|_{K^{\prime}} \leq Q(M)} h\left(x, x^{\prime}\right) \chi\left(N x / N^{\prime} x^{\prime}\right)_{K^{\times}}^{\times} x d_{K^{\prime}}^{\times} x^{\prime}\right) d x \\
=c^{\prime} e^{\prime \prime-1} \lim _{Q \rightarrow \infty} \int_{\left(x, x^{\prime}\right) \in B_{*},|N x| \leq Q} h\left(x, x^{\prime}\right) d_{B_{*}}^{\times}\left(x, x^{\prime}\right),
\end{array}\right.
$$

where $e^{\prime \prime}=e_{K^{\prime \prime} / F}$, and $h$ is now the function on $K^{\times} \times K^{\prime \times}$ given by

$$
h\left(x, x^{\prime}\right)=(\theta \psi \circ T)(x)\left(\theta^{\prime} \psi \circ T^{\prime}\right)\left(x^{\prime}\right)|N x|^{1 / 2}\left|N^{\prime} x^{\prime}\right|^{1 / 2} .
$$

We observe now that for $|\omega|<q$ and $\left|\omega^{\prime}\right|<q$, the function $h_{M}$ on $K^{\times} \times$ $K^{\prime \times}$ equal to $h$ on $|N x| \leq Q(M),\left|x^{\prime}\right|_{K^{\prime}} \leq Q(M)$, and 0 otherwise, lies in $\mathscr{S}\left(K^{\times} \times K^{\prime \times}\right)$. We apply Poisson summation formula (2:5.2) to this function $h_{M}:$

$$
\begin{aligned}
& \oint_{. \mathscr{G}\left(F^{\times}\right)}\left(\int_{K^{\times} \times K^{\prime \times}} h_{M}\left(x, x^{\prime}\right) \chi\left(N x / N^{\prime} x^{\prime}\right) d_{K}^{\times} x^{\prime}\right) d \chi \\
& =c_{1} \int_{B_{*}} h_{M}\left(x, x^{\prime}\right) d_{B_{*}}^{\times}\left(x, x^{\prime}\right) .
\end{aligned}
$$

On the left-hand side, only the characters $\chi$ with $a(\chi) \leq M$ will contribute, so this formula is

$$
\begin{aligned}
& \oint_{a(\chi) \leq M}\left(\int_{|N x| \leq Q(M),\left|x^{\prime}\right|_{K^{\prime}} \leq Q(M)} h\left(x, x^{\prime}\right) \chi\left(N x / N^{\prime} x^{\prime}\right) d_{K}^{\times} x d_{K^{\prime}}^{\times} x^{\prime}\right) d \chi \\
& =c_{1} \int_{\left(x, x^{\prime}\right) \in B_{*},|N x| \leq Q(M)} h\left(x, x^{\prime}\right) d_{B_{*}}^{\times}\left(x, x^{\prime}\right) .
\end{aligned}
$$

This clearly proves (3.1.7), and hence completes the proof of the theorem if we show $c_{1}=c^{\prime} e^{\prime \prime-1}$; but this is proved in the lemma of $\S 2.5$. 


\subsection{The multiplicative formula for $\gamma_{\theta}^{F}$.}

Theorem 2. Let $K$ be a quadratic étale $F$-algebra, and $\theta \in \mathscr{A}\left(K^{\times}\right)$. If $\alpha, \beta \in$ $\mathscr{A}\left(F^{\times}\right)$are not poles of $\chi \mapsto \gamma_{\theta}^{F}(\chi, \psi)$, then

$$
\begin{gathered}
\oint_{\mathscr{A}\left(F^{\times}\right)} \gamma_{\theta}^{F}(\chi, \psi) \Gamma\left(\alpha \chi^{-1}, \psi\right) \Gamma\left(\beta \chi^{-1}, \psi\right) d \chi \\
=\Gamma\left((\alpha \beta \omega)^{-1}, \psi^{-1}\right) \gamma_{\theta}^{F}(\alpha, \psi) \gamma_{\theta}^{F}(\beta, \psi)
\end{gathered}
$$

where $\omega=\left.\eta_{K / F} \theta\right|_{F^{\times}}$.

Proof. We apply Theorem 1 to $K^{\prime}=F \times F, \theta^{\prime}(u, v)=\alpha(u) \beta(v)|u v|^{-1 / 2}$. Then $B$ is $K \times K$ and

$$
\left(\theta \times \theta^{\prime}\right)(x y)=\theta(x y) \alpha(N x) \beta(N y)|N(x y)|^{-1 / 2},
$$

with $N=N_{K / F}$. As $\lambda_{B / F}=\lambda_{K / F}(\psi)^{2}$, using the complement formula in $\S 1.1$ for $\Gamma$, the right-hand side of $(3.1 .1)$ is written as

$$
|\psi| L_{F}^{-2} \Gamma\left((\alpha \beta \omega)^{-1}, \psi^{-1}\right) \gamma_{\theta}^{F}(\alpha, \psi) \gamma_{\theta}^{F}(\beta, \psi) \text {. }
$$

On the other hand,

$$
\begin{gathered}
\gamma_{\theta^{\prime}}^{F}\left(\chi^{-1}, \psi\right)=\gamma^{F}\left(\alpha q^{1 / 2} \chi^{-1}, \psi\right) \gamma^{F}\left(\beta q^{1 / 2} \chi^{-1}, \psi\right) \\
=\left(|\psi|^{1 / 2} L_{F}^{-1}\right)^{2} \Gamma\left(\alpha \chi^{-1}, \psi\right) \Gamma\left(\beta \chi^{-1}, \psi\right) .
\end{gathered}
$$

This gives (3.2.1).

3.3. Theorem 3. Let $K$ and $K^{\prime}$ be two nonisomorphic étale $F$-algebras, and let $B$ be their tensor product over $F$. Let $\theta$ and $\theta^{\prime}$ be two multiplicative characters of $K$ and $K^{\prime}$ respectively. Assume that the product of the restrictions of $\theta$ and $\theta^{\prime}$ to $F^{\times}$is trivial. Then, for any nontrivial additive character $\psi$ of $F$, one has

$$
\gamma^{B}\left(\theta \circ N_{B / K} \theta^{\prime} \circ N_{B / K^{\prime}}, \psi \circ T_{B / F}\right)=\theta(-1)=\theta^{\prime}(-1) .
$$

Proof. Write $\beta$ for the character $\theta \circ N_{B / K} \theta^{\prime} \circ N_{B / K^{\prime}}$ of $B^{\times}$. For $t$ in $F^{\times}$, one has

$$
\beta(t)=\theta\left(t^{2}\right) \theta^{\prime}\left(t^{2}\right)=\left(\theta \theta^{\prime}\right)\left(t^{2}\right)=1,
$$

hence $|\beta|=1$. The identity to prove is equivalent to the relation

$$
\int_{B^{\times}} \beta(z) \hat{f}(z) d_{B}^{*} z=\theta(-1) \int_{B^{\times}} \beta(z)^{-1} f(z) d_{B}^{*} z
$$

for any $f$ in $(B)$; the integrals are both convergent.

Denote by $K^{\prime \prime}$ the third quadratic $F$-algebra in $B$ determined by $K$ and $K^{\prime}$. Since $K$ and $K^{\prime}$ are not isomorphic, $K^{\prime \prime}$ is a field. By restriction to $K^{\prime \prime}$, the two automorphism groups of $B$ over $K$ and $K^{\prime}$ identify them with Gal $K^{\prime \prime} / F$. Hence, the norm maps $N_{B / K}$ and $N_{B / K^{\prime}}$ coincide on $K^{\prime \prime}$ with $N_{K^{\prime \prime} / F}$. As a consequence the restriction of $\beta$ to the multiplicative group of 
$K^{\prime \prime}$ is trivial. Denote by $d_{B, K^{\prime \prime}}^{*}$ the quotient measure of $d_{B}^{*}$ by $d_{K^{\prime \prime}}$, so, for $f \in \mathscr{S}(B)$,

$$
\int_{B^{\times}} \beta(z)^{-1} f(z) d_{B}^{*} z=\int_{B^{\times} / K^{\prime \prime \times}} \beta(z)^{-1}\left(\int_{K^{\prime \prime}} f(w z) d_{K^{\prime \prime}} w\right) d_{B, K^{\prime \prime}}^{*} z .
$$

We apply now the Poisson formula to the closed subgroup $K^{\prime \prime}$ of $B$ and to the function $f$

$$
\int_{K^{\prime \prime}} f(w z) d_{K^{\prime \prime}} w=|z|_{B}^{-1} \int_{K_{B}^{\prime \prime}} \hat{f}\left(z^{-1} w^{\prime}\right) d_{K_{B}^{\prime \prime}} w^{\prime},
$$

where $K_{B}^{\prime \prime}$ denotes the orthogonal of $K^{\prime \prime}$ in $B$ with respect to the self-duality $\left(z, z^{\prime}\right) \mapsto \psi \circ T_{B / F}\left(z z^{\prime}\right)$, that is, $K_{B}^{\prime \prime}=\operatorname{Ker} T_{B / K^{\prime \prime}}$, and where $d_{K_{B}^{\prime \prime}}$ denotes the Haar measure on $K_{B}^{\prime \prime}$ associated to $d_{K^{\prime \prime}}$. For $w^{\prime}$ in $K_{B}^{\prime \prime}$, one has $N_{B / K} w^{\prime}=$ $-N_{B / K^{\prime}} w^{\prime}$, hence the image of $K_{B}^{\prime \prime}$ under $N_{B / K}$ is contained in $K \cap K^{\prime}=F$. So if $w^{\prime} \in K_{B}^{\prime \prime}$ is not 0 , one has

$$
\beta\left(w^{\prime}\right)=\left(\theta \circ N_{B / K} w^{\prime}\right)\left(\theta^{\prime} \circ N_{B / K^{\prime}} w^{\prime}\right)=\theta(-1)=\theta^{\prime}(-1) .
$$

Choose a nonzero element, say $s$, in $K_{B}^{\prime \prime}$. We rewrite the right-hand side of (3.3.1) using (3.3.2)-(3.3.4) as

$$
\int_{B^{\times} / K^{\prime \prime x}} \beta\left(s z^{-1}\right)\left(\int_{K_{B}^{\prime \prime}} \hat{f}\left(z^{-1} w^{\prime}\right) d_{K_{B}^{\prime \prime}} w^{\prime}\right)|z|_{B}^{-1} d_{B, K^{\prime \prime}}^{*} z .
$$

We observe now that the measure $|z|_{B}^{-1} d_{B, K^{\prime \prime}}^{*} z$ is $d_{B, K^{\prime \prime}}^{*} z^{-1}$. We change the variables $w^{\prime}=s w, z \mapsto s z^{-1}$ in (3.3.5) to get

$$
\int_{B^{\times} / K^{\prime \prime \times}} \beta(z)\left(\int_{K^{\prime \prime}} \hat{f}(z w) d_{K^{\prime \prime}} w\right) d_{B, K^{\prime \prime}}^{*} z
$$

which is $\int_{B^{\times}} \beta(z) \hat{f}(z) d_{B}^{*} z$, the left-hand side of (3.3.1). This proves the theorem.

Corollary. With the same assumptions on $K$ and $K^{\prime}$, if now the product of the restrictions to $F^{\times}$of $\theta$ and $\theta^{\prime}$ is the character $q: t \mapsto|t|^{-1}$, then

$$
\left\langle\gamma_{\theta}^{F} \mid \gamma_{\theta^{\prime}}^{F}\right\rangle_{\psi}=\frac{\lambda_{B / K} \omega(-1)}{\Gamma\left(\eta_{K / F} \eta_{K^{\prime} / F} q^{-1}, \psi\right)},
$$

where $\omega(-1)=\theta(-1) \eta_{K / F}(-1)$.

Proof. This follows immediately from Theorems 1 and 3, due to the definition of $\lambda_{B / K}$ given in $\S 1.6$.

3.4. Theorem 4. Let $K$ and $K^{\prime}$ be two quadratic étale $F$-algebras, and $\theta \in$ $\mathscr{A}\left(K^{\times}\right), \theta^{\prime} \in \mathscr{A}\left(K^{\prime \times}\right)$. Then $\gamma_{\theta}^{F}=\gamma_{\theta^{\prime}}^{F}$ if and only if one of the following holds:

(1) for $K^{\prime}$ isomorphic to $K$, then $\theta^{\prime}$ corresponds to $\theta$ or to $\bar{\theta}$ by such an isomorphism; 
(2) for $K^{\prime}$ not isomorphic to $K$, let $B=K \otimes K^{\prime}$; then $\theta$ and $\theta^{\prime}$ have the same lifts to $B^{\times}$and different restrictions to $F^{\times}$.

Proof. If $K$ and $K^{\prime}$ are split, then $\gamma_{\theta}^{F}(\chi, \psi)=\gamma^{F}(\mu \chi, \psi) \gamma^{F}(\nu \chi, \psi)$ if $\theta=$ $\mu \otimes \nu$, and $\{\mu, \nu\}$ is determined by the zeros (or the poles), with multiplicities, of the function of $\gamma_{\theta}^{F}$. This establishes the theorem in this case.

Assume now $K$ is a field. If $\gamma_{\theta}^{F}=\gamma_{\theta^{\prime}}^{F}$, the deep twist property shows that $\omega=\omega^{\prime}$, hence $\left.\theta^{\prime}\right|_{F^{\times}}=\left.\eta_{K / F} \eta_{K^{\prime} / F} \theta\right|_{F^{\times}}$.

(a) If $K^{\prime}$ is not isomorphic to $K$, then $\eta_{K / F} \eta_{K^{\prime} / F}$ is nontrivial, since the third quadratic subalgebra of $B$ is a field.

(b) The equality $\gamma_{\theta}^{F}=\gamma_{\theta^{\prime}}^{F}$ implies $\gamma_{\theta^{-1}}^{F}(\chi, \psi)=\omega(-1) \gamma_{\theta^{\prime}}^{F}\left(\chi^{-1}, \psi\right)^{-1}$, so, as seen in $\S 2.3,\left\langle\gamma_{\theta^{-1}}^{F} \mid \gamma_{\theta^{\prime}}^{F}\right\rangle_{\psi}=0$. By Theorem 1, we then have

$$
\gamma^{B}\left(\theta^{-1} \circ N_{B / K} \theta^{\prime} \circ N_{B / K^{\prime}}, q_{B}^{-1 / 2}, \psi \circ T_{B / F}\right)=0 .
$$

(c) If $K$ and $K^{\prime}$ are isomorphic fields, then $B$ appears as $K \times K$ and $\gamma^{B}$ as product of two $\gamma^{K}$,s, so

$$
\gamma^{K}\left(\theta^{-1} \theta^{\prime} q_{K}^{-1 / 2}, \psi \circ T_{K / F}\right) \gamma^{K}\left(\theta^{-1} \bar{\theta}^{\prime} q_{K}^{-1 / 2}, \psi \circ T_{K / F}\right)=0
$$

which means $\theta^{\prime}=\theta$ or $\bar{\theta}$.

(d) If now $B$ is a field, then $\theta^{-1} \circ N_{B / K} \theta^{\prime} \circ N_{B / K^{\prime}}=1$, and $\theta$ and $\theta^{\prime}$ have the same lift to $B^{\times}$.

(e) If $K$ is a field and $K^{\prime}$ is $F \times F$, then $\theta^{\prime}=\mu \otimes \nu$ with $\mu, \nu \in \mathscr{A}\left(F^{\times}\right)$ and $B$ is $K \times K$; then

$$
\gamma^{K}\left(\mu \circ N_{K / F} \theta^{-1} q_{K}^{-1 / 2}, \psi \circ T_{K / F}\right) \gamma^{K}\left(\nu \circ N_{K / F} \theta^{-1} q_{K}^{-1 / 2}, \psi \circ T_{K / F}\right)=0,
$$

so $\theta$ is either $\mu \circ N_{K / F}$ or $\nu \circ N_{K / F}$. By the Davenport-Hasse theorem we have $\gamma_{\theta}^{F}(\chi, \psi)=\gamma^{F}(\mu \chi, \psi) \gamma^{F}\left(\mu \eta_{K / F} \chi, \psi\right)$ or $\gamma^{F}(\nu \chi, \psi) \gamma^{F}\left(\nu \eta_{K / F} \chi, \psi\right)$. As $\gamma_{\theta}^{F}(\chi, \psi)=\gamma_{\theta^{\prime}}^{F}(\chi, \psi)=\gamma^{F}(\mu \chi, \psi) \gamma^{F}(\nu \chi, \psi)$, we have $\mu \nu^{-1}=\eta_{K / F}$ in both cases; so $\theta=\mu \circ N_{K / F}=\nu \circ N_{K / F}$ and $\theta \circ N_{B / K}=(\mu \otimes \nu) \circ N_{B / K^{\prime}}$, which means that $\theta$ and $\theta^{\prime}$ have the same lift to $B^{\times}$.

We have proved the "necessary" part of the theorem. If $K$ and $K^{\prime}$ are isomorphic and $\theta^{\prime}$ corresponds to $\theta$ or $\bar{\theta}$, then $\lambda_{K / F}(\psi)=\lambda_{K^{\prime} / F}(\psi)$ and $\gamma_{\theta}^{F}=$ $\gamma \frac{F}{\theta}$ since both $\chi \circ N$ and $\psi \circ T$ are invariant by the conjugation of $K$ over $F$. Assume now $B$ is a field, and that $\theta \circ N_{B / K}=\theta^{\prime} \circ N_{B / K^{\prime}}$ with $\left.\theta\right|_{F^{\times}} \neq\left.\theta^{\prime}\right|_{F^{\times}}$. We use the third quadratic subextension $K^{\prime \prime}$ of $B$, and write $\theta \circ N_{B / K}=\theta^{\prime} \circ N_{B / K^{\prime}}$ on $K^{\prime \prime \times}$ : this shows that the restrictions to $N_{K^{\prime \prime} / F} K^{\prime \prime \times}$, which is an index two subgroup of $F^{\times}$, of $\theta$ and $\theta^{\prime}$ are equal. Hence $\left.\theta^{\prime}\right|_{F^{\times}}=\left.\eta_{K^{\prime \prime} / F} \theta\right|_{F^{\times}}$, that is, $\omega=\omega^{\prime}$. We prove that this implies that $\theta$ and $\theta^{\prime}$ are regular over $F$. Indeed, if $\theta=\mu \circ N_{K / F}$ then $\theta \circ N_{B / K}=\mu \circ N_{K^{\prime} / F} \circ N_{B / K^{\prime}}=\theta^{\prime} \circ N_{B / K^{\prime}}$; hence $\mu \circ N_{K^{\prime} / F}$ and $\theta^{\prime}$ coincide on the elements of $K^{\prime \times}$ which are norms 
from $B^{\times}$, in particular on $F^{\times}$: this contradicts $\left.\theta\right|_{F^{\times}} \neq\left.\theta^{\prime}\right|_{F^{\times}}$. The relation $\theta \circ N_{B / K}=\theta^{\prime} \circ N_{B / K^{\prime}}$ shows that this character of $B^{\times}$is fixed by both $\mathrm{Gal} B / K$ and $\mathrm{Gal} B / K^{\prime}$, hence by all $\mathrm{Gal} B / F$, that is, also by $\mathrm{Gal} B / K^{\prime \prime}$. This shows that $\theta$ and its conjugate $\bar{\theta}$ over $F$ have the same lift to $B^{\times}$, and $\theta$ being regular, this gives $\bar{\theta}=\theta \eta_{B / K}$, and also $\bar{\theta}^{\prime}=\theta^{\prime} \eta_{B / K^{\prime}}$. As $\eta_{B / K}$ is the lift of $\eta_{K^{\prime \prime} / F}$ to $K^{\times}$, the relation $\gamma_{\theta}^{F}=\gamma_{\theta}^{F}$ shows that $\gamma_{\theta}^{F}(\chi, \psi)=\gamma_{\theta}^{F}\left(\chi \eta_{K^{\prime \prime} \mid F}, \psi\right)$, and also for $\gamma_{\theta^{\prime}}^{F}$. We apply Theorem 1 to get the relation $\left\langle\gamma_{\theta^{\prime \prime}}^{F} \mid \gamma_{\theta}^{F}\right\rangle_{\psi}=\left\langle\gamma_{\theta^{\prime \prime}}^{F} \mid \gamma_{\theta^{\prime}}^{F}\right\rangle_{\psi}$ for $\theta^{\prime \prime} \in \mathscr{A}\left(K^{\prime \prime \times}\right)$, with a finite number of exceptions. We take $\theta^{\prime \prime}=\chi \circ N_{K^{\prime \prime} \mid F}$ and use the Davenport-Hasse identity to get

$$
\gamma_{\theta}^{F}(\chi, \psi) \gamma_{\theta}^{F}\left(\chi \eta_{K^{\prime \prime} / F}, \psi\right)=\gamma_{\theta^{\prime}}^{F}(\chi, \psi) \gamma_{\theta^{\prime}}^{F}\left(\chi \eta_{K^{\prime \prime} / F}, \psi\right),
$$

that is, $\gamma_{\theta}^{F}(\chi, \psi)^{2}=\gamma_{\theta^{\prime}}^{F}(\chi, \psi)^{2}$. We define a sign $\varepsilon(\chi)$ by

$$
\gamma_{\theta^{\prime}}^{F}(\chi, \psi)=\varepsilon(\chi) \gamma_{\theta}^{F}(\chi, \psi), \quad \chi \in \mathscr{A}\left(F^{\times}\right) .
$$

This sign is 1 for $\chi$ with large conductor, due to the deep twist property and $\omega=\omega^{\prime}$. As $\theta$ and $\theta^{\prime}$ are regular, the rational functions $\gamma_{\theta}^{F}(\chi, \psi)$ and $\gamma_{\theta^{\prime}}^{F}(\chi, \psi)$ are monomials on $\mathscr{A}\left(F^{\times}\right)$, and having the same squares, the degree is the same, as $\varepsilon(\chi)$ is constant on each component of $\mathscr{A}\left(F^{\times}\right)$. Theorem 1 shows that $\left\langle\gamma_{\theta^{\prime}}^{F} \mid \gamma_{\theta^{-1}}^{F}\right\rangle_{\psi}=0$, hence, for $M$ large enough,

$$
\begin{aligned}
0 & =\oint_{a(\chi) \leq M} \gamma_{\theta^{\prime}}^{F}(\chi, \psi) \gamma_{\theta^{-1}}^{F}\left(\chi^{-1}, \psi\right) d \chi-q^{M}\left(1-q^{-1}\right) \\
& =\left(\oint_{a(\chi) \leq M} \varepsilon(\chi) d \chi-q^{M}\left(1-q^{-1}\right)\right) \omega(-1),
\end{aligned}
$$

that is, $\sum_{a(\chi) \leq M} \varepsilon(\chi)=\sum_{a(\chi) \leq M} 1$, the summations being on the characters of $\mathscr{O}^{\times}$with $a(\chi)$ at most $M$. As $\varepsilon(\chi)$ is a sign, this implies $\varepsilon(\chi)=1$ for all $\chi$ 's, and $\gamma_{\theta}^{F}=\gamma_{\theta^{\prime}}^{F}$. The theorem is completely proved.

Corollary. With $K, K^{\prime}, \theta, \theta^{\prime}$ as in the theorem, we have $\gamma_{\theta}^{F}=\gamma_{\theta^{\prime}}^{F}$ if and only if $\operatorname{Ind}_{K}^{F} \theta=\operatorname{Ind}_{K^{\prime}}^{F} \theta^{\prime}$.

Proof. (a) This is clear if $K$ and $K^{\prime}$ are isomorphic since $\operatorname{Ind}_{K}^{F} \theta$ and $\operatorname{Ind}_{K^{\prime}}^{F} \theta^{\prime}$ are the same if and only if $\theta^{\prime}=\theta$ or $\bar{\theta}$, which means $\gamma_{\theta}^{F}=\gamma_{\theta^{\prime}}^{F}$ by Theorem 4.

(b) Assume $K$ is a field and $K^{\prime}$ is $F \times F$. Write $\theta^{\prime}=\mu \otimes \nu$, so that $\operatorname{Ind}_{K^{\prime}}^{F} \theta^{\prime}$ is the direct sum of the two one-dimensional representations $\mu$ and $\nu$ of $F^{\times}$, abelianized group of $W_{F}$. Theorem 4 shows that $\gamma_{\theta}^{F}=\gamma_{\theta^{\prime}}^{F}$ if and only if $\theta=\mu \circ N_{K / F}$ and $\nu=\mu \eta_{K / F}$; then $\operatorname{Ind}_{K}^{F} \theta=\mu \otimes \operatorname{Ind}_{K}^{F} 1=\mu \oplus \mu \eta_{K / F}=$ $\mu \oplus \nu=\operatorname{Ind}_{K^{\prime}}^{F} \mu \otimes \nu=\operatorname{Ind}_{K^{\prime}}^{F} \theta^{\prime}$. Conversely, if $\operatorname{Ind}_{K}^{F} \theta$ is the sum of the two characters $\mu$ and $\nu$, then $\operatorname{Ind}_{K}^{F} \theta$ is not irreducible, so $\theta=\chi \circ N_{K / F}$; but then $\operatorname{Ind}_{K}^{F} \theta=\chi \oplus \chi \eta_{K / F}$ so that $\chi=\mu$ or $\nu$ and $\nu=\mu \eta_{K / F}$, and the theorem says $\gamma_{\theta}^{F}=\gamma_{\theta^{\prime}}^{F}$. 
(c) Assume now $B=K \otimes K^{\prime}$ is a field. If $\gamma_{\theta}^{F}=\gamma_{\theta^{\prime}}^{F}$, then, by Theorem 4, we have $\theta \circ N_{B / K}=\theta^{\prime} \circ N_{B / K^{\prime}}$ and $\left.\theta\right|_{F^{\times}} \neq\left.\theta^{\prime}\right|_{F^{\times}}$; during the proof, we have shown that this implies $\bar{\theta}=\theta \eta_{B / K}$ and $\bar{\theta}^{\prime}=\theta^{\prime} \eta_{B / K^{\prime}}$. As the trace of $\operatorname{Ind}_{K}^{F} \theta$ is 0 outside $W_{K}$ and on $W_{K}$ it factors through its abelianization $K^{\times}$where it is given by $(\theta+\bar{\theta}) / 2$, this is 0 outside $W_{B}$ and on $W_{B}$ it factors through its abelianization $B \times$ where it is given by $\theta \circ N_{B / K}$. Hence $\operatorname{Ind}_{K}^{F} \theta$ and $\operatorname{Ind}_{K^{\prime}}^{F} \theta^{\prime}$ have their traces supported on $W_{B}$, where they are equal, so the representations $\operatorname{Ind}_{K}^{F} \theta$ and $\operatorname{Ind}_{K^{\prime}}^{F} \theta^{\prime}$ are equivalent. Conversely, this equivalence implies that traces and determinants of two representations are the same; for the determinants, this gives $\left.\theta\right|_{F} \times \eta_{K / F}=\left.\theta^{\prime}\right|_{F^{\times}} \eta_{K^{\prime} / F}$, so $\left.\theta\right|_{F^{\times}}$and $\left.\theta^{\prime}\right|_{F^{\times}}$are different; for the traces, we get 0 outside $W_{K} \cap W_{K^{\prime}}=W_{B}$, that is, $\bar{\theta}=\theta \eta_{B / K}, \bar{\theta}^{\prime}=\theta^{\prime} \eta_{B / K^{\prime}}$, and the coincidence on $W_{B}$ says that $\theta \circ N_{B / K}=\theta^{\prime} \circ N_{B / K^{\prime}}$. Theorem 1 then concludes the proof.

\section{REFERENCES}

[D] P. Deligne, Les constantes des équations fonctionnelles des fonctions $L$, Modular Functions in One Variable. II, Lecture Notes in Math., vol. 349, Springer-Verlag, Berlin-HeidelbergNew York, 1973, pp. 501-597.

[G] P. Gérardin, Groupes quadratiques et applications arithmétiques, Séminaire de Théorie des Nombres, Paris, 1984-85 (C. Golstein, Ed.), Progress in Math., Vol. 63, Birkhäuser, Boston, Mass., 1986, pp. 79-95.

[GK] P. Gérardin and P. C. Kutzko, Facteurs locaux pour GL(2), Ann. Sci. École Norm Sup. (4) 13 (1980), 359-384.

[GL1] P. Gérardin and W.-C. W. Li, A functional equation for degree two local factors, Canad. Math. Soc. Bull. 28 (1985), 355-371.

[GL2] __ Fourier transforms of representations of quaternions, J. Reine Angew. Math. 349 (1985), 121-173.

[GL3] _ Degree two monomial representations of local Weil groups, J. Reine Angew. Math. 394 (1989), 1-30.

[L] W.-C. W. Li, Barnes' identities and representations of GL . Part II: Nonarchimedean local field case, J. Reine Angew. Math. 345 (1983), 69-92.

[S] J.-P. Serre, Corps locaux, Hermann, Paris, 1964.

[W1] A. Weil, Sur certains groupes d'opérateurs unttaires, Acta Math. 111 (1964), 153-211.

[W2] _ Basic number theory, Springer-Verlag, Berlin and New York, 1967.

[W3] _ Fonctions zeta et distributions, Séminaire Bourbaki, Juin 1966.

Department of Mathematics, Pennsylvania State University, University Park, PennSYLVANIA 16802 